\title{
Flexible RSV prefusogenic fusion glycoprotein exposes multiple neutralizing epitopes that may collectively contribute to protective immunity
}

\author{
Nita Patel' ${ }^{1}$, Jing-Hui Tian', Rhonda Flores ${ }^{1}$, Kelsey Jacobson' ${ }^{1}$, Michelle Walker ${ }^{1}$, Alyse Portnoff ${ }^{1}$, Mimi \\ Gueber-Xabier', Michael J. Massare', Greg Glenn' ${ }^{1}$, Larry Ellingsworth', and Gale Smith ${ }^{1 *}$ \\ ${ }^{1}$ Novavax, Inc. 21 Firstfield Road, Gaithersburg, MD, 20878, USA. npatel@novavax.com (N.P.), jhtian@Novavax.com

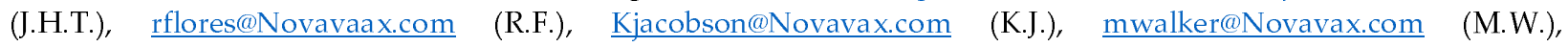 \\ aportnoff@Novavax.com (A.P), mguebre-xabier@Novavax.com (M.G.X.), MMassare@Novavax.com (M.J.M.), \\ gglenn@Novavax.com (G.G.), lellingsworth@novavax.com (L.E.), GSmith@Novavax.com (G.S.)
}

*Correspondence: GSmith@Novavax.com (G.S.)

\begin{abstract}
Human respiratory syncytial virus (RSV) is a significant cause of lower respiratory tract infection in infants, young children, and older adults. There is no licensed vaccine and prophylactic treatment options are limited and not widely available in developing countries with the greatest disease burden. The RSV fusion (F) glycoprotein is a primary target of host immunity and thus a major focus for vaccine development. The native $\mathrm{F}$ glycoprotein structure is flexible and undergoes significant rearrangements from the metastable prefusion to a stable postfusion structure with neutralizing epitopes on intermediate structures. We hypothesize trimeric vaccine strategies that recapitulate the breathable $\mathrm{F}$ quaternary structure, and provide accessibility of B-cells to epitopes on intermediate conformations, may collectively contribute to protective immunity, while ridge prefusion F structures restrict access to key protective epitopes. To test this hypothesis, we used the near full-length native prefusogenic $\mathrm{F}$ as a backbone to construct three prefusion F variants with substitutions in the hydrophobic head cavity: 1) disulfide bond double mutant (DS), 2) space filling hydrophobic amino acid substitutions (Cav1), and 3) DS plus Cav1 substitutions (DS-Cav1). In this study, we compared the immunogenicity of prefusogenic F to the immunogenicity of the prefusion F variants in two animal models. Native prefusogenic F was significantly more immunogenic producing high titer antibodies to prefusogenic, prefusion, and postfusion F structures compared to animals immunized with prefusion F DS or DS-Cav1. Importantly, native prefusogenic $\mathrm{F}$ elicited antibodies that targeted neutralizing epitopes including prefusion-specific site zero $(\varnothing)$ and $\mathrm{V}$ as well as conformation-independent neutralizing sites II and IV. Immunization with prefusion F DS or DS-Cav1 elicited antibodies primarily targeting antigenic sites $\varnothing$ and V with little or no detectable antibodies to other key neutralizing sites. Animals immunized with native prefusogenic $\mathrm{F}$ also had significantly higher neutralizing antibodies that cross-neutralized RSV A and B subtypes while immunization with DS or DS-Cav1 elicited neutralizing antibodies primarily to the A subtype. We conclude that breathable trimeric vaccines that closely mimic the native F-structure, and incorporate strategies for B-cell accessibility to protective epitopes, are important considerations for vaccine design. F structures locked in a single conformation restrict B-cell access to neutralizing epitopes that may collectively contribute to destabilizing F-trimers important for broad protection. These results also have implications for vaccine strategies targeting other type 1 integral membrane proteins.
\end{abstract}

Keywords: respiratory syncytial virus; fusion glycoprotein; prefusogenic RSV F; cotton rat 


\section{Introduction}

Human respiratory syncytial virus (RSV) is a cause of lower respiratory infection (LRTI) in young children and older adult populations. The disease burden is particularly high in developing countries with over 3 million hospitalizations and 50,000-70,000 deaths in young children. Children under 5 years of age are the most susceptible and account for $45 \%$ of RSV-related deaths with the vast majority (>90\%) in developing countries [1]. RSV disease burden is also substantial in older adults with over 1 million infections, 300,000 hospitalizations, and over 10,000 in-hospital deaths [2]. Although RSV is the cause of significant worldwide disease burden, there are no licensed vaccines and palivizumab (Synagis ${ }^{\circledR}$ ) is the only licensed prophylaxis for prevention of RSV in high-risk newborn. There is no established correlate of protective immunity [3-5].

RSV is a negative-strained, enveloped RNA virus in the Pneumoviridae family. The fusion (F) glycoprotein is a major component of the virus envelope. RSV F protein is conserved between human RSV A and B subtypes ( $>90 \%$ amino acid identity) with shared neutralizing epitopes, is essential for infection, and a major target of host immune defense [6-10]. RSV F is a type 1 integral membrane protein produced as a $70 \mathrm{kDa}$ inactive precursor (F0). Unique to RSV, the F glycoprotein has two cleavage sites at positions R109 (site I) and R136 (site II) that are processed by host cell furin-like proteases. Removal of the intervening 27 amino acid peptide (p27) generates a small F2 subunit and a larger F1 subunit [11-14]. The F1 subunit contains the fusion peptide (FP) on the N-terminus, two heptad repeats (HRA and HRB), and the transmembrane (TM) domain and cytoplasmic tail (CT) on the C-terminus. The F2 subunit contributes to fusogenicity, and contains a single heptad repeat (HRC) [7,15-18]. F2/F1 are covalently linked by 2disulfide bonds to form a protomer. Three F2/F1 protomers associate through weak non-covalent interprotomeric bonds to form the functional F-trimer. Trimers are highly flexible and transiently open and close, dissociate, and monomerize within the lipid bilayer exposing a range of conformations recognized by B-cell receptors $[19,20]$.

Prefusion F undergoes significant rearrangements to a stable postfusion F during attachment and fusion. Events triggering F-protein rearrangement are not understood, although elevated temperatures promote spontaneous rearrangement of the heptad repeats (HRA, HRB and HRC) to form the fusogenic six-helix bundle (6HB) that releases the FP from the hydrophobic cavity. FP insertion into the host membrane is essential for alignment of the virus and host cell membranes, fusion, pore formation, and release of the virus genome into the host cell [21]. RSV F variants with amino acid substitutions in the hydrophobic cavity stabilize F in the prefusion conformation. Disulfide-bond double mutant DS (S155C and S290C), Cav1 with cavity filling hydrophobic amino acids at positions S150F and V207L, and DSCav1 with four substitutions have been extensively characterized and shown to bind neutralizing monoclonal antibodies (mAbs) [22,23]. It has been proposed that prefusion $\mathrm{F}$ is a superior vaccine candidate since antibodies bind antigenic sites zero $(\varnothing)$ and $V$ with high affinity, although there is no clinical evidence supporting protective efficacy [24].

Monoclonal antibodies (mAbs) from convalescent patients have provided insight into the plasticity of the humoral immune response to subtle conformational changes in the F structure. For instance, human neutralizing mAbs D25, AM22, and RSD5 all bind prefusion-specific antigenic site $\varnothing$ with high affinity [22,25]. Crystal structures of prefusion DS (PDB: 4MMQ) and DS-Cav1 (PDB: 4MMS), in complex with D25, AM22, and RSD5 Fabs (fragment antigen binding), show these Fabs bind site $\varnothing$ at 3 distinct angles [26,27]. Gillman et al. [19] have recently compared binding of human mAbs CR9501 and hRSV90 to antigenic site V. Crystal structures of CR9501 Fab in complex with antigenic site V shows the Fab is rotated by $60^{\circ}$ compared to hRSV90 and CDR makes contact with F2. Significantly, CR9501 binding 
destabilizes F-trimers and promotes disassembly [19]. Similarly, mAbs palivizumab and motavizumab bind sites II at distinct angles and with different affinity $[9,28,30]$. We recently reported that murine $\mathrm{mAb}$ R4.C6 binds a quaternary epitope consisting of antigenic site II on one protomer and site IV on a neighboring protomer [28]. Collectively, these observations demonstrate that B-cells recognize a wide range of intermediate trimeric and monomeric $\mathrm{F}$ structures. These observations and others suggest trimeric vaccines candidates that mimic the breathable native $\mathrm{F}$ structure, allowing B-cell accessibility to sequestered epitopes, not only block receptor binding but, more importantly, may destabilize the trimeric structure.

We have described the development of a RSV prefusogenic F constructed from the near full-length RSV/A2 F protein in which furin cleavage site II was mutated to retain the native $\mathrm{p} 27$ with a truncated FP $(\triangle \mathrm{FP})$ on the N-terminus of F1. F1/F2 protomers assemble as trimers and 3-6 F-trimers spontaneously associate to form $35-40 \mathrm{~nm}$ nanoparticles. Prefusogenic F nanoparticles are immunogenic and protective in the cotton rats [29,31], elicit maternal antibodies that protect newborn infant baboons against pulmonary RSV challenge [32], and is safe and immunogenic in women of childbearing age (NCT02624947) [33], and older adults [34]. Here, we used prefusogenic F as a backbone to assess the effects of introducing prefusion stabilizing mutations (DS, Cav1, DS-Cav1) upon the immunogenicity of prefusogenic F. Overall we find that prefusogenic F was significantly more immunogenic than ridged DS or DS-Cav1 variants. Importantly, immunization with prefusogenic F elicited a broad range of antibodies that bound prefusion-specific antigenic sites $\varnothing$ and $V$ as well as serotype conserved neutralizing sites II and IV. Prefusion DS and DS-Cav1 variants were less immunogenic and elicited antibodies primarily to sites $\varnothing$ and $\mathrm{V}$ with little or no antibodies to other neutralizing $\mathrm{F}$ epitopes. These findings suggest breathable $\mathrm{F}$ vaccine candidates produce broad range of antibodies to neutralizing sites that may collectively contribute to protection against RSV. These results have implications for vaccine strategies targeting other type 1 integral membrane proteins.

\section{Materials and Methods}

\subsection{Cell Lines, Viruses, Synthetic peptides, and Monoclonal Antibodies (mAbs)}

HEp-2 (ATCC, CCL-23, Manassas, VA, USA) cells were maintained in MEM with Earle's salts and Lglutamine (Gibco Laboratories, Gaithersburg, MD, USA), 10\% fetal bovine serum (FBS; HyClone, Logan, UT, USA), and antibiotics (Life Technologies, Grand Island, NY, USA). RSV/A Long challenge stock (lot KJ060319, $2.21 \times 10^{7} \mathrm{pfu} \mathrm{mL}^{-1}$ ) was propagated in HEp-2 cells and produced by Sigmovir Inc. (Gaithersburg, MD, USA). Synthetic p27 peptide (RARRELPRFMNYTLNNPKKTNVTLSKKPKRRF) was produced by Thermo Fischer, Inc. Palivizumab (site II) was obtained from MedImmune, Inc. (Rockville, MD, USA). hRSV90 (site VIII also referred to as site V) [35] was provided Dr. James Crowe (Vanderbilt Vaccine Center, Vanderbilt University, Nashville, TN). D25 (site Ø) $[8,22,23]$ was purchased from Creative BioLabs (Shirley, NY, USA). Mouse mAbs R1.42 (site IV) and R7.10 (p27) were produced by Novavax, Inc. [28,31]. World Health Organization (WHO) human RSV Reference Standard was purchased from the National Institute of Biological Standards and Control (NIBSC code 16/284, 2000 IU $\mathrm{mL}^{-1}$, Potters Bar, Hertfordshire, UK).

Other reagents. RSV F DS-Cav1-293 reagent was produced by transient transfection of HEK293 T cells with pcDNA 3580 encoding the prefusogenic F gene with the DS (S55C and S290C) and the Cav1 (S190F and V207L) mutations. HEK293 T cells were transfected with lipofectamine 3000 following the manufacturer's procedure (Invitrogen, Carlsbad, CA, USA). Prefusion F (BV2129) reagent was 
constructed from the prefusogenic F (BV1184) backbone with stabilizing triple mutations at positions N67I, S215P and E487Q [17] and produced in Spodoptera frugiperda (Sf9) insects cells.

\subsection{RSV F Glycoprotein Constructs}

Synthetic RSV F genes were cloned into the pFasBac1 (Invitrogen) downstream of the AcMNPV polyhedron promoter (GeneArt, Regensburd, DE, USA). RSV F transgenes were cloned into recombinant baculovirus (BV) using the Bac-to-Bac BV system [29]. Sf9 cells (Invitrogen, Grand Island, NY, USA) were cultured in serum-free medium. RSV F proteins were constructed from the near full-length RSV/A2 F gene sequence (Genbank Accession No. U63644) encoding 574 amino acids. The constructs were codon optimized for expression in insect cells. To produce the prefusogenic F construct (BV1184), furin cleavage site II was mutated (KKRKRR $\rightarrow \mathrm{KKQKQQ)}$ ) to be protease resistant and the adjacent 10 amino acids deleted (F137-V146) from the fusion peptide $(\triangle \mathrm{FP})$ on the N-terminus of the $\mathrm{F} 1$ subunit and the native transmembrane (TM) and cytoplasmic tail (CT) retained on the C-terminus of the F1 subunit (GenBank Accession No. MN125707) $[29,31,36]$. Three prefusion F constructs were generated using the prefusogenic F (BV1184) backbone: (1) RSV F DS (BV2267) was produced by introducing disulfide bond double mutations (DS) at positions S155C and S290C within the F1 subunit; (2) RSV F Cav1 (BV2279) construct was generated by introducing hydrophobic amino acid substitutions at positions S190F and V207L; and (3) RSV F DS-Cav1 (BV2280) was constructed with the DS and Cav1 substitutions [22]. Postfusion F (BV2128) was constructed with the native furin cleavage sites I and II and the truncated fusion peptide $(\triangle \mathrm{FP})$. N-terminal TM and CT domains were deleted and replaced with a 6-histidine tag $(6-\mathrm{H})[30,31]$.

\subsection{Purification of RSV F Proteins}

Prefusogenic F (BV1184), postfusion F (BV2129), prefusion F DS (BV2267), Cav1 (BV2279), and DSCav1 (BV2280) were produced by infection of Sf9 cells (MOI $=0.5 \mathrm{pfu} / \mathrm{cell}$ ) with recombinant BV for 67 hours at $27^{\circ} \mathrm{C}$. Cells were harvested by centrifugation and RSV F proteins extracted from detergent lysed cell membranes with $25 \mathrm{mM}$ Tris-HCL (pH 8.0), $50 \mathrm{mM} \mathrm{NaCl}, 0.5 \%(\mathrm{v} / \mathrm{v})$ Tergitol ${ }^{\mathrm{TM}} \mathrm{NP}-9$, and $2.0 \mu \mathrm{g} \mathrm{mL}^{-1}$ leupeptin (Sigma-Aldrich, St. Louis, MO, USA). RSV F proteins were purified by a combination of anion exchange, lentil lectin affinity, and cation ion exchange chromatography [29]. For soluble postfusion $\mathrm{F}$ (BV2128), Sf9 cells were infected with recombinant BV for 67 hours and supernatants were clarified by centrifugation. RSV F (BV2128) was purified with an immobilized metal affinity column (IMAC) and ion exchange chromatography [31]. Purified RSV F proteins $\left(0.33-1.5 \mathrm{mg} \mathrm{mL}^{-1}\right)$ were formulated in $25 \mathrm{mM}$ phosphate buffer (pH 6.8), $150 \mathrm{mM} \mathrm{NaCl}, 0.032 \%$ (v/v) polysorbate- 80 (PS80), and 1\% (w/v) histidine.

\subsection{Antigenic Site-specific mAb Binding to RSV F Proteins Determined by ELISA}

Site-specific mAbs binding to recombinant RSV $\mathrm{F}$ proteins was determine by ELISA. Purified prefusogenic F (BV1184) and prefusion F proteins DS (BV2267), Cav1 (BV2279), DS-Cav1 (BV2280), and DS-Cav1-293 were coated $\left(2.0 \mu \mathrm{g} \mathrm{mL}^{-1}\right)$ in 96 well micro titer plates overnight at $4^{\circ} \mathrm{C}$. Following coating, the plates were blocked with 2\% milk blocking buffer (Quality Biologicals, Gaithersburg, MD, USA). Primary mAbs D25 (site Ø), hRSV90 (site V), palivizumab (site II), R1.42 (site IV), R7.10 (p27) were serially diluted $\left(1.0 \mu \mathrm{g} \mathrm{mL}^{-1}\right.$ to $\left.0.01 \mathrm{ng} \mathrm{mL}^{-1}\right)$. WHO human RSV reference antiserum (RSV Ref Std) was serially diluted $10^{-2}$ to $10^{-8}$. Plates were incubated for 2 hours at room temperature and washed with phosphate buffered saline containing $0.05 \%$ Tween (PBS-T). Horseradish peroxidase (HRP)-conjugated goat anti-human IgG (2040-05, Southern Biotech) or HRP-conjugated goat anti-mouse IgG (1030-05, Southern Biotech) second antibodies were added to the wells. After 1 hour, the wells were washed with PBS-T and 3,3',5,5'-tetramethylbenzidine peroxidase substrate (TMB, T0440-IL, Sigma, St Louis, MO, 
USA) added to the wells. Reactions were stopped with TMB stop solution (TSB999, Scytek Lab, Logan, UT, USA). Plates were read at an optical density (OD) $450 \mathrm{~nm}$ with a SpectraMax plus plate reader (Molecular Devices, Sunnyvale, CA, USA). EC50 values were calculated by 4-parameter fitting using Prism 7.05 software.

\subsection{Design of Animal Studies}

Animal ethics statement. Noble Life Sciences (Sykeville, MD, USA) performed the murine immunogenicity study. Noble Life Sciences is accredited by the Association for Assessment and Accreditation of Laboratory Animal Care (AAALAC International). Sigmovir Biosystems, Inc. (Rockville, MD, USA) performed the cotton rat immunogenicity and RSV challenge study. Sigmovir is licensed by the United States Department of Agriculture (USDA permit 51-R-0091) and the Office of Laboratory Animal Welfare (OLAW permit A4642-01). The cotton rat study was conducted in accordance with appropriate sections of the National Research Council Guide for the Care and Use of Laboratory Animals and the Animal Welfare Act Regulations. Both studies were performed in accordance with Institutional Animal Care and Use Committee (IACUC) approved protocols.

Murine study design. Female BALB/c mice (7-9 weeks old, 17-22 grams, $\mathrm{N}=10$ per group) were immunized by intramuscular (IM) injection with $1.0 \mu \mathrm{g}$ of RSV F prefusogenic F, prefusion $\mathrm{F}$ variants (DS, Cav1, DS-Cav1) or postfusion F adjuvanted with $30 \mu \mathrm{g}$ aluminum phosphate (Adju-Phos ${ }^{\circledR}$, Brenntag Biosector, Frederikssund DK) administered in 2-doses spaced 21-days apart (day 0, 21). A separate group $(\mathrm{N}=10)$ received a single intranasal (IN) challenge with $20 \mu \mathrm{L}$ of $10^{6} \mathrm{pfu}$ RSV/A Long (10 $\mu \mathrm{L}$ each nare) on study day 0 . Serum was collected for analysis from individual animals on study day 35 (14-days after the second immunization).

Cotton rat study design. Female cotton rats (4-6 weeks old, 50-65 grams) were randomly assigned to groups ( $\mathrm{N}=6$ per groups) and immunized by IM injection with $1.0 \mu \mathrm{g}$ of the indicated RSV F protein with $30 \mu \mathrm{g}$ aluminum phosphate (lot 9496, Brenntag Biosector) administered in 2-doses spaced 21-days apart (day 0,21). A control group $(\mathrm{N}=6$ ) received placebo (non-immunized) and was used as a positive RSV challenge control. A separate group $(\mathrm{N}=6)$ received a single IN challenge with $100 \mu \mathrm{L}$ of $10^{6} \mathrm{pfu}$ RSV/A Long divided between each nare on study day 0. Serum was collected via the orbital plexus 21days after the second immunization (day 42) and stored at $-20^{\circ} \mathrm{C}$ until analyzed. Animals were challenged with $100 \mu \mathrm{L}$ of $10^{5} \mathrm{pfu}$ RSV/A Long (50 $\mu \mathrm{L}$ each nare) 21-days after the second immunization (day 42). Nose and lung homogenates were prepared 4-days post-challenge (day 46) and virus load determined by a plaque assay.

\subsection{Anti-RSV F Glycoproteins IgG ELISA}

An ELISA was used to determine anti-RSV F IgG titers. Briefly, 96 well microtiter plates (Thermo Fischer Scientific, Rochester, NY, USA) were coated with $2.0 \mu \mathrm{g} \mathrm{mL}^{-1}$ of prefusogenic F (BV1184), prefusion F (BV2129), DS-Cav1 (BV2280), or postfusion F (BV2128). Plates were washed with PBS-T and blocked with blocking buffer (Quality Biologicals, Gaithersburg, MD, USA). Serum samples were serially diluted $\left(10^{-2}\right.$ to $\left.10^{-8}\right)$ and the plates incubated at room temperature for 2 hours. Following incubation, plates were washed with PBS-T and HRP-conjugated goat anti-mouse IgG or anti-rat IgG (Southern Biotech, Birmingham, AL, USA) added for 1 hour. Plates were washed with PBS-T and TMB peroxidase substrate (T0440-IL, Sigma, St Louis, MO, USA) added. Reactions were stopped with TMB stop solution (ScyTek Laboratories, Inc. Logan, UT). Plates were read at OD $450 \mathrm{~nm}$ with a SpectraMax plus plate reader (Molecular Devices, Sunnyvale, CA, USA) and data analyzed with SoftMax software. EC50 values 
were calculated by 4-parameter fitting using GraphPad Prism 7.05 software. Individual animal anti-RSV F protein IgG titers and group geometric mean titers (GMT) and $95 \%$ confidence interval $( \pm 95 \% \mathrm{CI})$ were plotted.

\subsection{RSV Neutralizing Assay}

Mouse and cotton rat serum samples were serially diluted in 96 well microtiter plates. Palivizumab and human RSV reference standard (NIBSC 16/284, Potters Bar, Hertfordshire, UK) were used to generate standard curves. RSV/A Long or RSV/B 18537 (TCID50 $=200-350$ ) was added to the wells and incubated for 2 hours at $37^{\circ} \mathrm{C}$ in $5 \% \mathrm{CO}_{2}$. Following adsorption, wells were seeded with $2.5 \times 10^{4} \mathrm{HEp}-2$ cells and cultured for 4-days at $37^{\circ} \mathrm{C}$ in $5 \% \mathrm{CO}_{2}$. After incubation, the cultures were washed and air dried. To detect RSV infection, the cultures were treated with mouse monoclonal (RSV5H5) anti-RSV M2-1 (ab94805, Abcam, Cambridge, MA, USA). HRP-conjugated goat anti-mouse IgG second antibody (catalog number 1030-05, Southern Biotech, Birmingham, AL, USA) was added. TMB substrate was added to the wells and the plates read at OD $450 \mathrm{~nm}$ with a plate reader. Neutralizing titers were calculated relative to the human RSV reference standard $\left(2000 \mathrm{IU} \mathrm{mL}^{-1}\right)$ or palivizumab standard (4600 $\left.\mathrm{IU} \mathrm{mL}^{-1}\right)$ using a 4parameter fit using GraphPad Prism 7.05 software. Individual animal titers were plotted with the group GMT $\pm 95 \%$ CI indicated.

\subsection{Prefusion and Postfusion F Specificity of Antibodies Determined by RSV F Competeted ELISA}

Levels of serum antibodies specific for prefusion and postfusion F structures was determined with a competitive ELISA. Briefly, 96 well plates were coated with $2.0 \mu \mathrm{g} \mathrm{mL}^{-1}$ prefusion F (BV2129) overnight at $4^{\circ} \mathrm{C}$. Immune cotton rat serum or RSV reference antiserum was 3-fold serially diluted and mixed (1:1 ratio) with $20 \mu \mathrm{g} \mathrm{mL}^{-1}$ of prefusion F (BV2129) or postfusion F (BV2128) for 1 hour at room temperature. Following incubation, $100 \mu \mathrm{L}$ of serum/protein mixture was transferred to the prefusion $\mathrm{F}$ coated microtiter wells. The mixture was incubated for 2 hours at room temperature and the wells and washed with PBS-T. HRP conjugated goat anti-rat IgG or anti-human IgG was added followed by addition of TMB substrate. Plates were read at OD $450 \mathrm{~nm}$ with plate reader and the EC50 values calculated by 4parameter curve fitting with GraphPad Prism software.

\subsection{Prefusion and Postfusion F Specificity of Neutralizing Antibodies Determined by Competitive ELISA}

Levels of neutralizing antibodies bound by prefusion and postfusion F structures was determined with a competitive ELISA. Cotton rat serum was serially diluted in 96 well microtiter plates and $5 \mu \mathrm{gL}^{-1}$ prefusion $\mathrm{F}$ (BV2129) or postfusion $\mathrm{F}$ (BV2128) added for 1 hour at $37^{\circ} \mathrm{C}$ followed by addition of RSV/A (TCID50 $=200-300$ ) for 2 hours at $37^{\circ} \mathrm{C}$. Following incubation, $2.5 \times 10^{4} \mathrm{Hep} 2$ cells were added to the wells and the plates incubated at $37^{\circ} \mathrm{C}$ and $5 \% \mathrm{CO}_{2}$ for 4 days. After incubation, the cultures were washed and air dried. Virus replication was detected with mouse mAb RSV5H5 anti-RSV M2-1 (ab94805, Abcam, Cambridge, MA, USA) followed by addition of HRP-conjugated goat anti-mouse IgG second antibody (catalog number 1030-05, Southern Biotech, Birmingham, AL, USA). TMB substrate was added and the plates read at OD $450 \mathrm{~nm}$ with a plate reader. Percent neutralization was calculated compared to the virus control cultures (no serum).

\subsection{Antigenic Site-Specific Antibodies Determined Antibody ELISA}

A competitive antibody binding assay was used to determine the levels of antibodies in mouse and cotton rat serum that competed the binding of RSV F site-specific mAbs [29]. Briefly, monoclonal antibodies D25 (site Ø), palivizumab (site II), R1.42 (site IV), and R7.10 (p27) were biotinylated using the 
manufacturers protocol (Pierce, Rockford, IL, USA). For sites II, IV and p27 determinations, microtiter plates were coated with $2.0 \mu \mathrm{g} \mathrm{mL}^{-1}$ prefusogenic RSV F protein (BV1184). For site $\varnothing$ determinations, plates were coated with $2.0 \mu \mathrm{g} \mathrm{mL}^{-1}$ prefusion RSV F protein (BV2129). After overnight incubation, the plates were washed with PBS-T and blocked with $2 \%$ milk blocking buffer for 1 hour at room

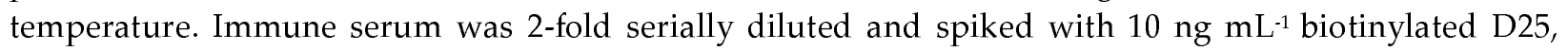
palivizumab, R1.42, or $50 \mathrm{ng} \mathrm{mL}^{-1} \mathrm{R} 7.10$. Biotinylated antibodies without immune serum was used as a no competition control. After 2 hours at room temperature, the plates were washed with PBS-T and $100 \mu \mathrm{L}$ diluted HRP conjugated Streptavidin (e-Bioscience, San Diego, CA, USA) was added for 1 hour and plates washed with PBS-T. TMB substrate was added, color developed, and reaction stopped with TMB stopping buffer. Plates were read at OD $450 \mathrm{~nm}$ with plate reader. Data was analyzed with Softmax pro software (Molecular Devices). Competing antibody concentration was determined from the RSV reference standard curve by interpolating the concentration of the reference standard that corresponds to $50 \%$ competition. Antigenic site-specific antibody levels $\left(\mu \mathrm{g} \mathrm{mL}^{-1}\right)$ were plotted for individual animals and the group GMT $\pm 95 \% \mathrm{CI}$ indicated.

\subsection{Plaque Assay}

Nose and lung tissue homogenates were prepared from cotton rats 4-days following IN challenge with RSV/A Long. Homogenates were clarified by centrifugation. Confluent HEp-2 monolayers were infected in duplicate with $50 \mu \mathrm{L}$ per well of serially diluted homogenate samples in 24 -well plates. After 1 hour at $37^{\circ} \mathrm{C}$ in $5 \% \mathrm{CO}_{2}, 0.75 \%$ methylcellulose medium overlay added and the cells cultured for 6-days. Cells were fixed with $10 \%$ neutral formalin and stained with $0.01 \%$ crystal violet solution $(1.5 \mathrm{~mL} /$ well $)$. Plaques were counted and titers expressed as $\mathrm{pfu} \mathrm{g}^{-1}$ of nasal or lung homogenate. Virus titers were plotted for individual animals and the group GMT $\pm 95 \% \mathrm{CI}$ indicated.

\subsection{Statistical Analysis}

Statistical comparisons between paired groups was performed with a student t-test and one-way ANOVA using Graph Pad Prism 7.05 software.

\section{Results}

\subsection{RSV F Glycoproteins Proteins}

We have described a prefusogenic RSV F (BV1184) vaccine candidate constructed from the near fulllength RSV/A2 F-glycoprotein that form nanoparticles consisting of 3-5 F-trimers [29,31]. Using the prefusogenic $\mathrm{F}$ as a backbone, prefusion constructs were produced by introducing mutations within the hydrophobic cavity to stabilized the prefusion F structure as described by McLellan et al. $[22,23]$. RSV F DS (BV2267) was constructed with the disulfide bond double mutation (DS) at positions S155C and S290C; RSV F Cav1 (BV2279) with amino acid substitutions at positions S190F and V207L; and RSV F DSCav1 (BV2280) with the DS and Cav1 mutations (Figure 1A and 1B). 
A
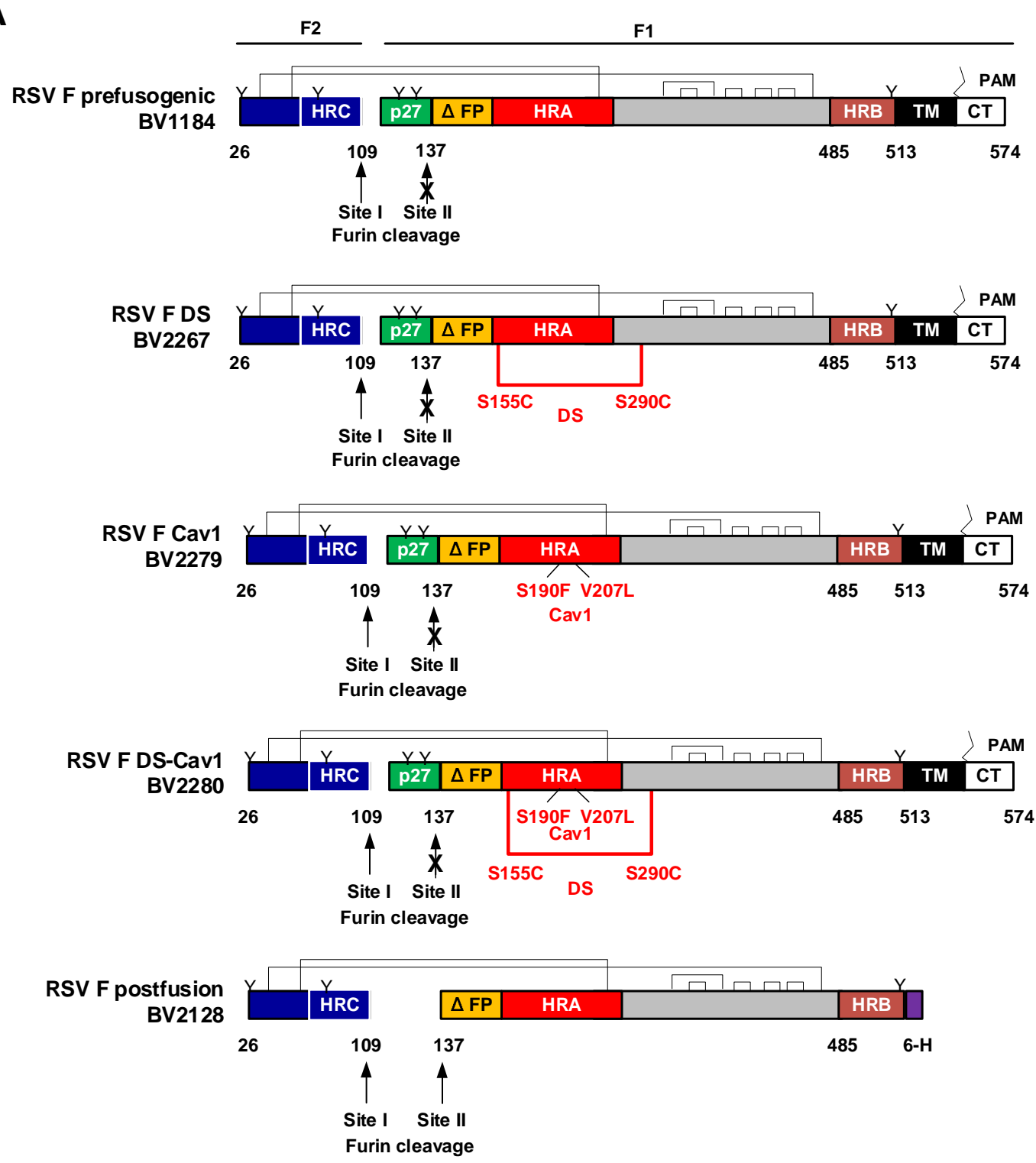

B

Prefusogenk F

Prefuslon F

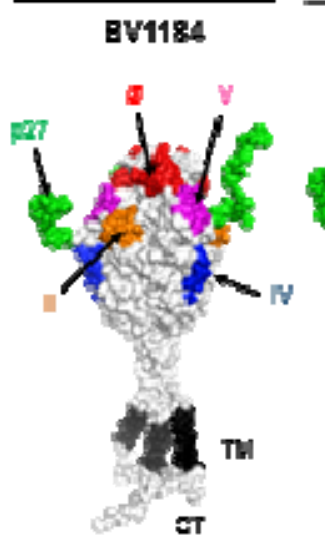

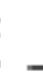

Dg
8v2267
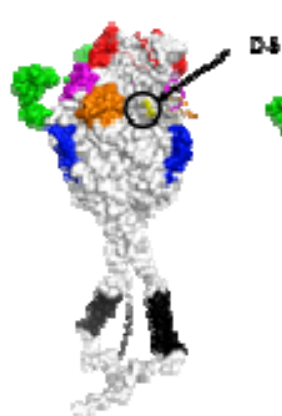

$\operatorname{cov} 1$

BV2778

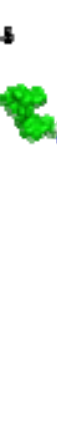

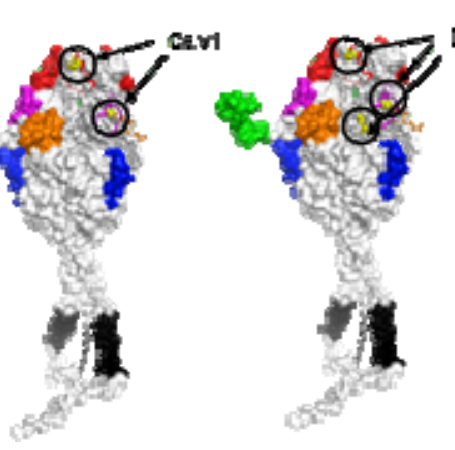

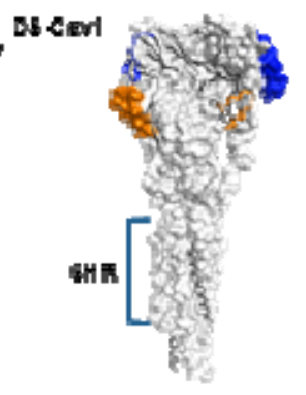

\section{Postfus on $F$}

BV2128 
Figure 1. RSV F constructs used in this study. (A) Linear diagram of the RSV F glycoproteins constructed from the wild-type RSV/A2 fusion glycoprotein gene sequence (GenBank accession number MN125707). RSV F has two furin cleavage sites (arrows) that flank the intervening 27 amino acid (p27 fragment, green). To produce the prefusogenic RSV F (BV1184), furin cleavage site II was mutated (KKRKRR $\rightarrow$ KKQKQQ) to be protease resistant and is retained on the N-terminus of the F1 subunit. The first 10 amino acids of the adjacent fusion peptide were deleted $(\triangle \mathrm{FP}$, yellow). The native C-terminal native transmembrane (TM, black) and cytoplasmic tail (CT, white) were retained on the N-terminus of F1. The resulting F2 and F1 ectodomain is covalently coupled by two disulfide bonds (brackets). $N$-linked

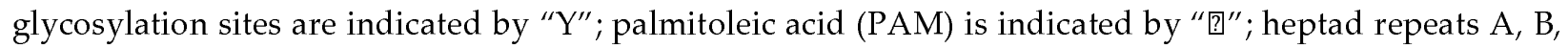
and $C$ are indicated by HRA (red), HRB (brown) and HRC (blue). The prefusogenic F structure (BV1184) backbone was used to produce three prefusion F variants. RSV F double mutant (DS) was generated by introducing a stabilizing disulfide bond at positions S155C and S290C within the F1 subunit (designated BV2267). RSV F Cav1 (BV2279) construct was generated by introducing two hydrophobic amino acids at positions S190F and V207L. The RSV F DS-Cav1 construct (BV2280) was generated with S155C/S290C disulfide bond and the S190F/V207L substitutions. Postfusion F (BV2128) was generated with intact furin cleavage sites I and II and truncation of the fusion peptide ( $\triangle \mathrm{FP}$, yellow). The TM and CT domains were deleted and replaced with a 6-histidine tag (6-H, purple). (B) Surface structures of the RSV F prefusogenic and prefusion trimers indicating key neutralizing antigenic sites antigenic sites $\varnothing$ (red), V (pink), II (orange), and IV (blue), and the p27 fragment (green). The native transmembrane (TM, black) and cytoplasmic tail (CT, grey) are indicated. The RSV F postfusion 6-heptarepeat bundle (6HR) is indicted.

\subsection{Antigenic Site-specific Binding of $m A$ bs to RSV F Proteins by ELISA}

A panel of human and murine mAbs targeting RSV F neutralizing sites was used to characterize the antigenic profile of the RSV F constructs. Using an ELISA method, prefusion-stable RSV F DS (BV2267), Cav1 (BV2279), DS-Cav1 (BV2280), and DS-Cav1-293 were bound by prefusion-specific mAbs D25 (site $\varnothing)$ and hRSV90 (site V) at low antibody concentrations (ED50 $=1.07-1.66 \mathrm{ng} \mathrm{mL}^{-1}$ ), indicating neutralizing sites $\varnothing$ and V were intact on these prefusion constructs. Prefusogenic F nanoparticles (BV1184) also by bound by mAbs D25 and hRSV90, although at higher antibody concentrations (ED50 $=47.40 \mathrm{ng} \mathrm{mL}^{-1}$ and $26.80 \mathrm{ng} \mathrm{mL}^{-1}$, respectively) suggesting these prefusion sites are present on prefusogenic $\mathrm{F}$, although at lower levels (Figure 2A and 2B). Humanized palivizumab [9] and murine R1.42 mAbs [28] were used to detect antigenic sites II and IV, respectively. Prefusogenic F and prefusion F variants (DS, Cav1, DS-Cav1 and DS-Cav1-293) bound palivizumab (ED50 = 1.0-3.0 $\left.\mathrm{ng} \mathrm{mL}^{-1}\right)$ and R1.42 (ED50 = 2.4-6.1 $\mathrm{ng} \mathrm{mL}^{-1}$ ) at low antibody concentrations indicating sites II and IV were present on prefusogenic $\mathrm{F}$ and the prefusion $\mathrm{F}$ variants (Figure 2C and 2D). Prefusogenic F and prefusion DS, Cav1, and DS-Cav1 variants also bound mAb R7.10 at low concentration (ED50 $=2.7-3.17 \mu \mathrm{g} \mathrm{mL}^{-1}$ ) consistent with the $\mathrm{p} 27$ being preset on these F-trimers. DS-Cav1-293 bound R7.10 at a higher concentration (ED50 $=22.1 \mu \mathrm{g} \mathrm{mL}^{-1}$ ) compared to the other constructs, suggesting p27 may be less available to antibody binding (Figure 2E). All RSV F structures bound WHO RSV reference antibodies equally (EC50 $=1.12 \mathrm{E}-04$ to 3.97E-04) (Figure 2F). These results indicate that antigenic sites $\varnothing, \mathrm{V}, \mathrm{II}, \mathrm{IV}$, and $\mathrm{p} 27$ were present on prefusogenic $\mathrm{F}$ and the DS, Cav1, DS-Cav1 and DS-Cav-293 variants. 

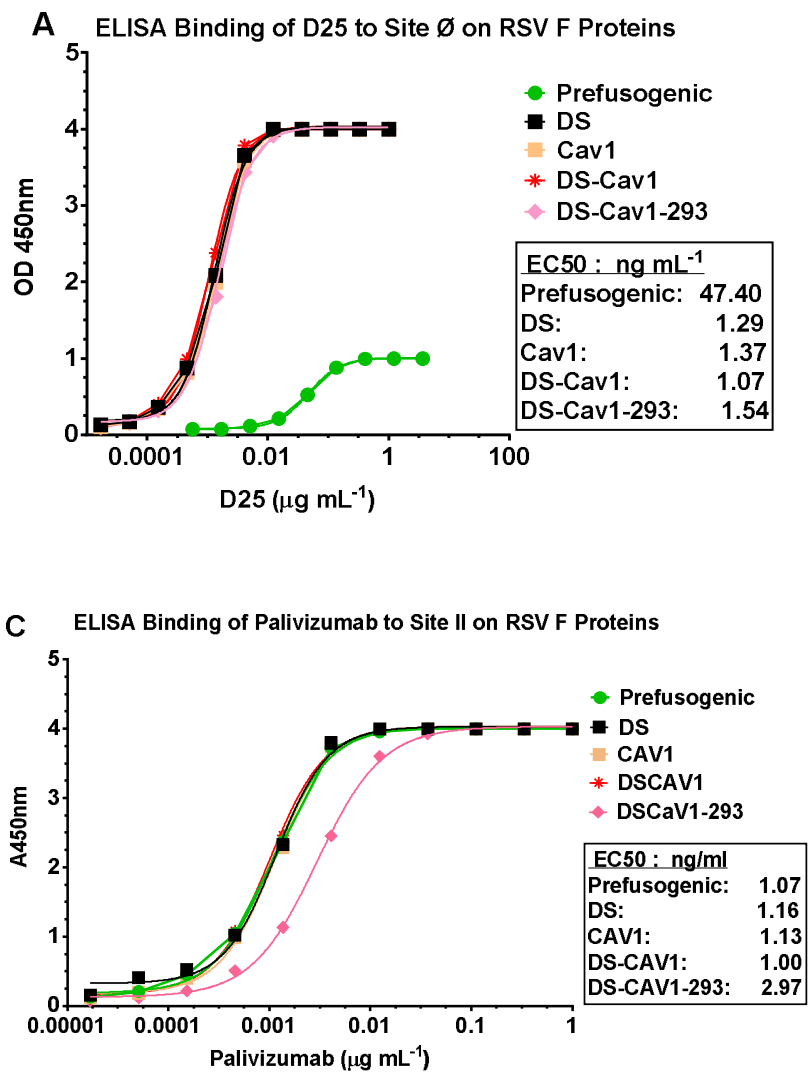
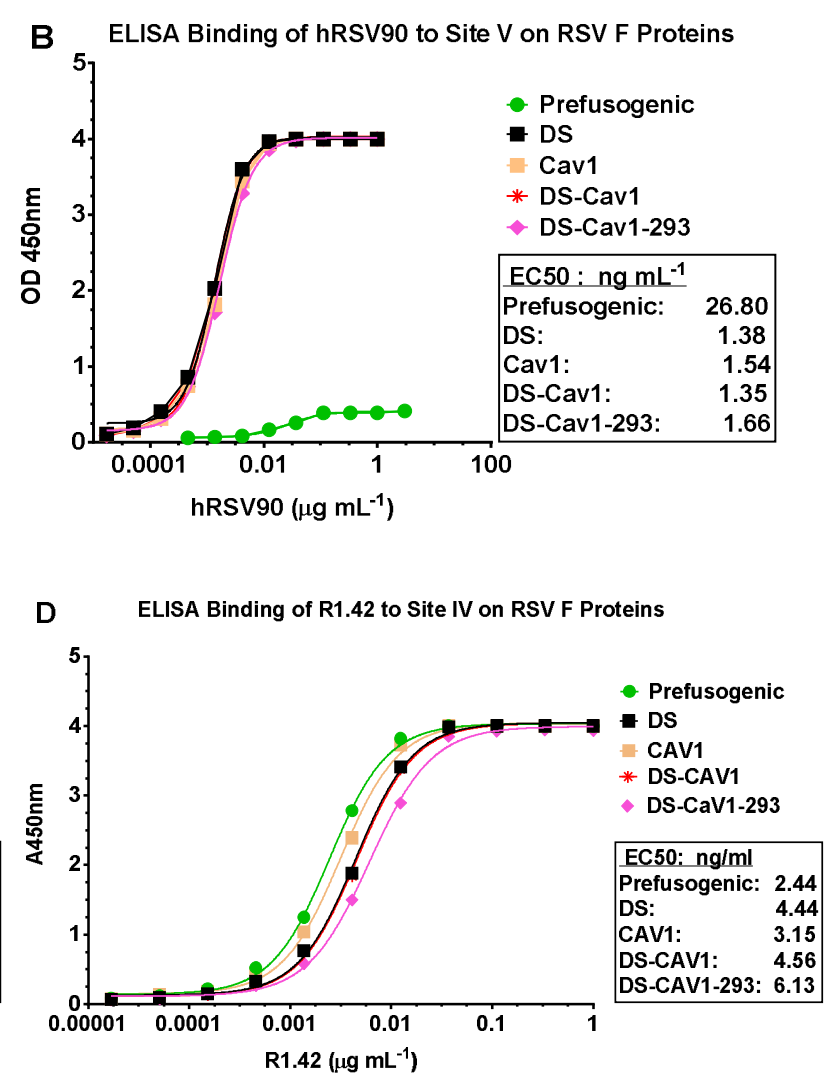
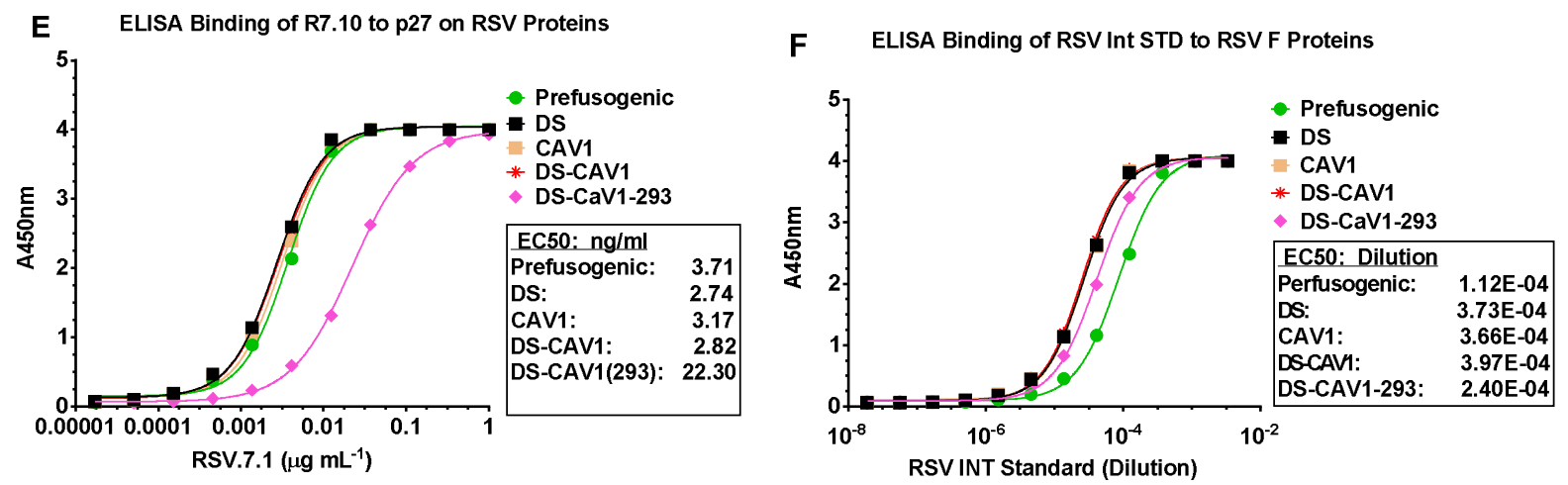

Figure 2. Antigenic site-specific monoclonal antibody binding to prefusogenic $F$ and prefusion $F$ variants. ELISA binding curves (A) D25 (site Ø), (B) hRSV90 (site V), (C) palivizumab (site II), (D) R1.42 (site IV), (E) R7.10 (p27), and (F) RSV international standard (RSV Int Std).

\subsection{Immunogenicity of RSV F Proteins in Mice}

The immunogenicity of prefusogenic F was compared to prefusion DS, Cav1, and DS-Cav1 F variants in mice. Animals immunized with prefusogenic F (BV1184) had significantly higher $(\mathrm{p}<0.0001)$ antibody titers to prefusogenic F BV1184 (GMT = 384,547) compared to immunization with DS (GMT = 2736) or DSCav1 $($ GMT $=1038)$. Animals immunized with prefusogenic F also had significant higher $(\mathrm{p} \leq 0.0007)$ 
antibody titers to prefusion F BV2129 (GMT $=223,670)$ compared to animals immunized with prefusion F variants DS (GMT $=20,527$ ) or DS-Cav1 (GMT = 19,195) (Figure 3A and 3B). Immunized with Cav1 (BV2279) also elicited high titer antibodies to prefusogenic F BV1184 (GMT $=161,953)$ and prefusion F BV2129 (GMT = 109,422) that were not significantly different from prefusogenic F (Figure 3A and 3B).

Serum antibodies targeting neutralizing epitopes was determined by competitive ELISA using sitespecific mAbs. Mice immunized with Cav1 had the highest levels of antibodies (GMT $=7.8 \mu \mathrm{g} \mathrm{mL}^{-1}$ ) competitive with D25 (site Ø), while serum from mice immunized with prefusogenic F, DS, or DS-Cav1 variants had significantly lower $(\mathrm{p} \leq 0.034)$ levels of antibodies that competed D25 binding (GMT $=0.7-1.4$ $\left.\mu \mathrm{g} \mathrm{mL}{ }^{-1}\right)$. These results suggest that antigenic site $\varnothing$ was poorly immunogenic in mouse regardless of the immunogen (Figure 3C).

Mice immunized with prefusogenic $F$ and Cav1 had significantly higher $(p<0.0001)$ levels of antibodies that competed the binding of palivizumab to site II (GMT $=620.0$ and $224.5 \mu \mathrm{g} \mathrm{mL}^{-1}$, respectively), while mice immunized with prefusion F DS or DS-Cav1 had little or no antibodies targeting site II (GMT $=4.0$ and $2.5 \mu \mathrm{g} \mathrm{mL}-1$, respectively) (Figure 3D). Similarly, animals immunized with prefusogenic F or Cav1 had significantly $(\mathrm{p}<0.0001)$ elevated levels of antibodies that competed binding of R1.42 to site IV (GMT = 676.9 and $204.456 .0 \mu \mathrm{g} \mathrm{mL}^{-1}$, respectively), while animals immunized with DS or DS-Cav1 had no detectable antibodies that competed binding of R1.42 to site IV (Figure 3E). Animals immunized with postfusion $\mathrm{F}$ also had low levels of antibodies competitive with palivizumab for site II $\left(\mathrm{GMT}=33.8 \mu \mathrm{g} \mathrm{mL}^{-1}\right)$ and variable levels of antibodies competitive with R1.42 for site IV (GMT $=46.7 \mu \mathrm{g}$ $\mathrm{mL}^{-1}$ ) (Figure 3D and 3E).

Antibody to p27 were also determined. Mice immunized with prefusogenic F or Cav1 had significantly higher $(\mathrm{p}<0.0001)$ levels of antibodies that competed with R7.10 to the $\mathrm{p} 27$ peptide (GMT $=$ 112.2 and $80.9 \mu \mathrm{g} \mathrm{mL}^{-1}$, respectively) (Figure 3F). In contrast, immunization with DS or DS-Cav1 variants elicited no measurable antibodies to 27 (GMT $<0.35 \mu \mathrm{g} \mathrm{mL}^{-1}$ ) suggesting that p27 was not accessible to Bcells in DS and DS-Cav1 stabilized F (Figure 3F). Animals immunized with the prefusogenic F, DS, or DS-Cav1 variants had similar levels of RSV/A Long neutralizing antibodies (GMT $=86.2$ to $225.4 \mathrm{IU} \mathrm{mL}^{-1}$ ) (Figure 3G). Immunization with postfusion F (BV2128) produced significantly lower ( $p=0.011$ ) levels of neutralizing antibodies. Taken together, these results demonstrate that wild-type prefusogenic $\mathrm{F}$ was significantly more immunogenic than prefusion F DS or DS-Cav1. Antigenic site $\varnothing$ was poorly immunogenic and elicited little or no antibodies competitive with D25 in mice regardless of the immunogen. Prefusogenic F and Cav1 were equally immunogenic and elicited antibodies targeting sites II and IV while immunization with prefusion stable DS and DS-Cav1 had little or no antibodies to antigenic sites II or IV or p27, suggesting these sites were not accessible to humoral immune response on the prefusion-stable F structures. 

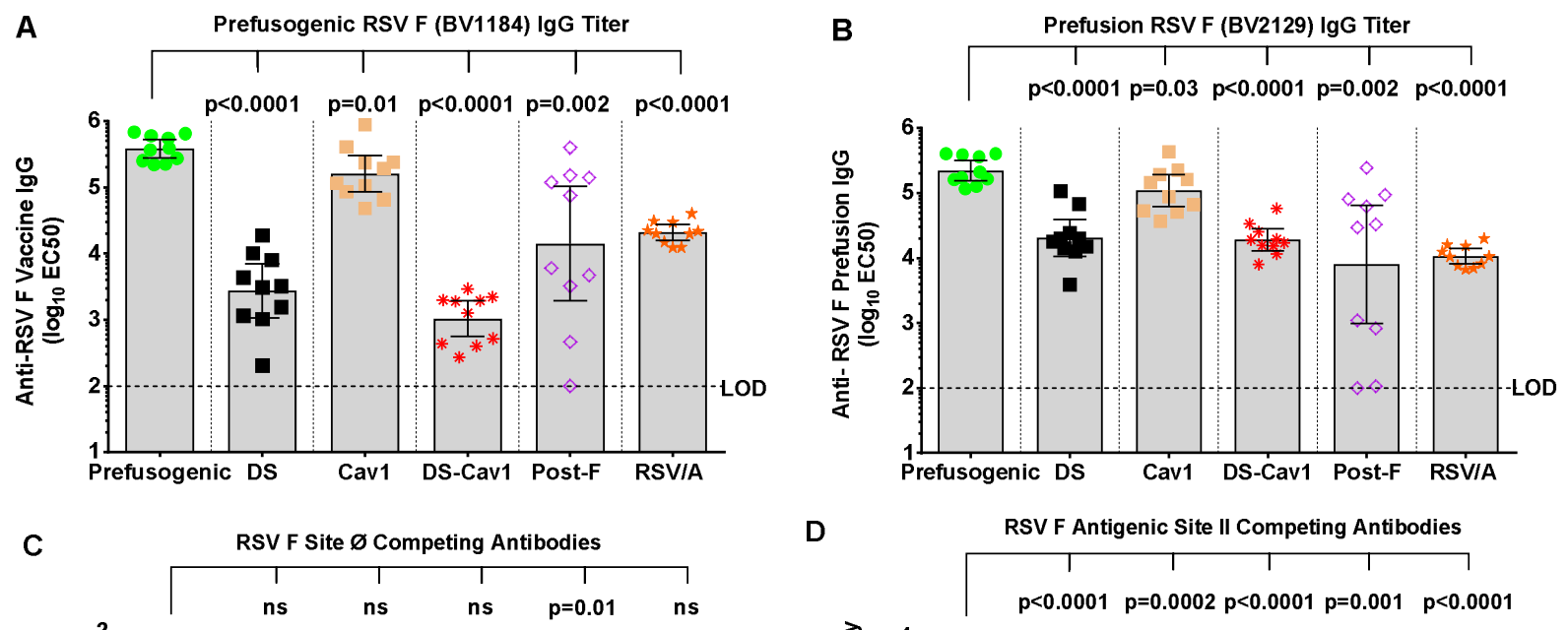

D
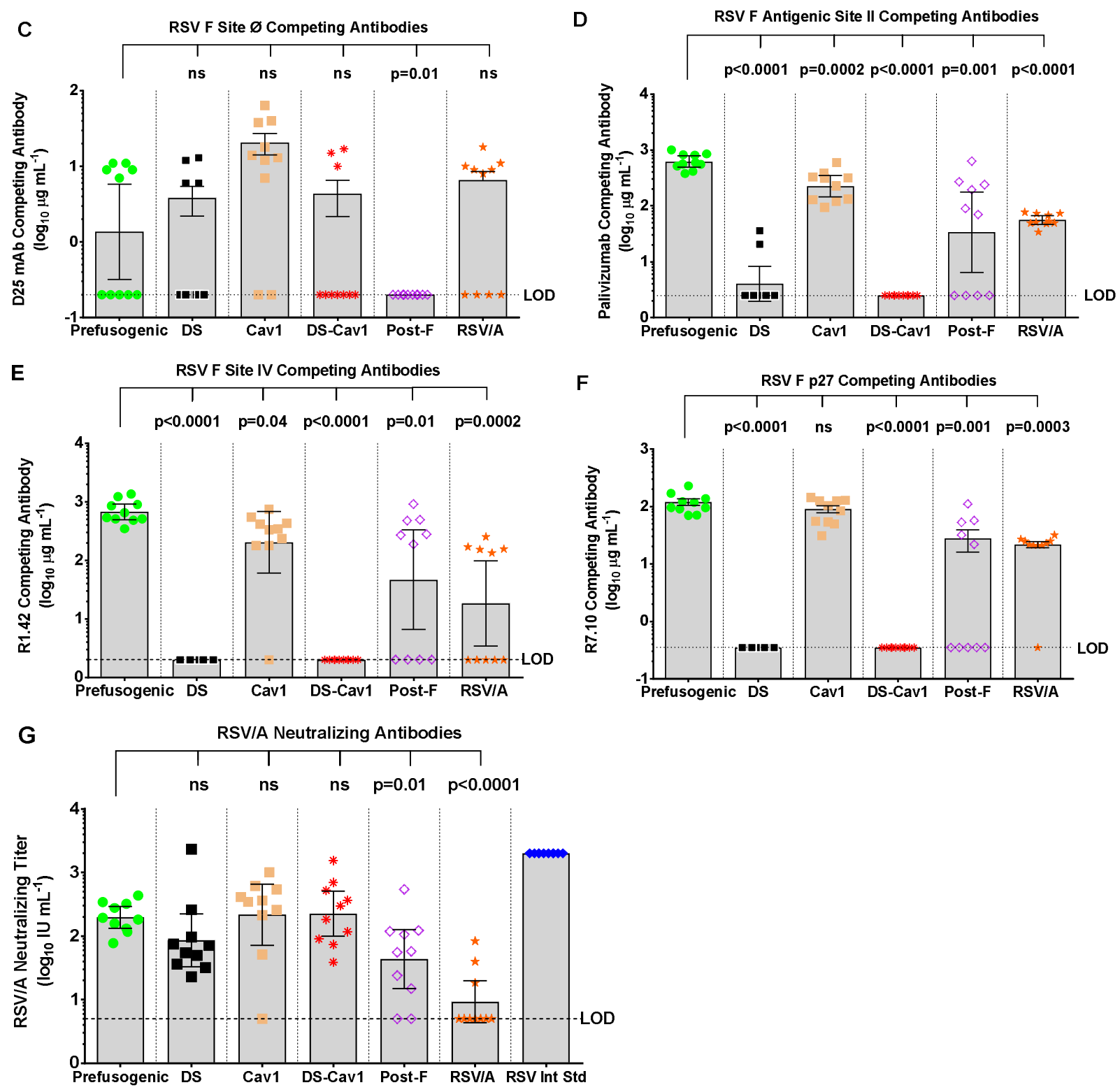
Figure 3. Immunogenicity of prefusogenic $\mathrm{F}$ and $\mathrm{F}$ variants in mice. Groups of mice were immunized by intramuscular injection with prefusogenic F vaccine (BV1184), DS (BV2267), Cav1 (BV2279), DS-Cav1 (BV2280) or postfusion F (BV2128) adjuvanted on days 0 and 21. A separate group was challenged intranasal with $10^{6} \mathrm{pfu}$ RSV/A Long. Serum was collected for analysis from all animals on study day 35 (14-days after the second immunization). (A) Anti-prefusogenic F vaccine IgG (BV1184) and (B) antiprefusion F IgG (BV2129) determined by ELISA. A competitive antibody ELISA was used to determine serum levels of antibodies that competed the binding of site-specific mAbs (C) D25 (site Ø), (D) palivizumab (site II), (E) R1.42 (site IV), and (F) R7.10 (p27). (G) RSV neutralizing antibody titers were determined by inhibition of HEp-2 cell infection with RSV/A Long. The horizontal bars indicate the group ( $N=10$ per group) geometric mean titer (GMT) and the error bars indicate the $95 \%$ confidence interval ( $\pm 95 \%$ CI). Significance between the RSV F prefusogenic vaccine (BV1184) group and paired RSV F protein immunized groups is indicated. Limit of detection (LOD). Not significant (ns).

\subsection{Immunogenicity of RSVF Proteins in Cotton Rats}

The immunogenicity of prefusogenic and prefusion $\mathrm{F}$ variants was also compared in cotton rats. Animals immunized with prefusogenic $F$ had significantly higher $(p<0.0001)$ titer antibodies to prefusogenic F BV1184 (GMT $=285,691)$ compared to animals immunized with DS (GMT $=18,361)$ or DSCav1 (GMT = 4590) (Figure 4A). Animals immunized with prefusogenic F also had significantly higher $(\mathrm{p}<0.0001)$ antibody titers to postfusion F BV2128 (GMT $=164,082)$ compared to DS or DS-Cav1 (GMT = 3424 and 2177, respectively) (Figure 4B). Interestingly, animals immunized with prefusogenic F and DS, Cav1, or DS-Cav1 had equivalent antibody titers to prefusion F (BV2129) and DS-Cav1 (BV2280) (Figure $4 \mathrm{C}$ and $4 \mathrm{D}$ ). These results demonstrate that prefusogenic $\mathrm{F}$ elicited high titer antibodies to prefusion and postfusion F structures while cotton rats immunized with DS and DS-Cav1 produced antibodies primarily targeting prefusion structures.
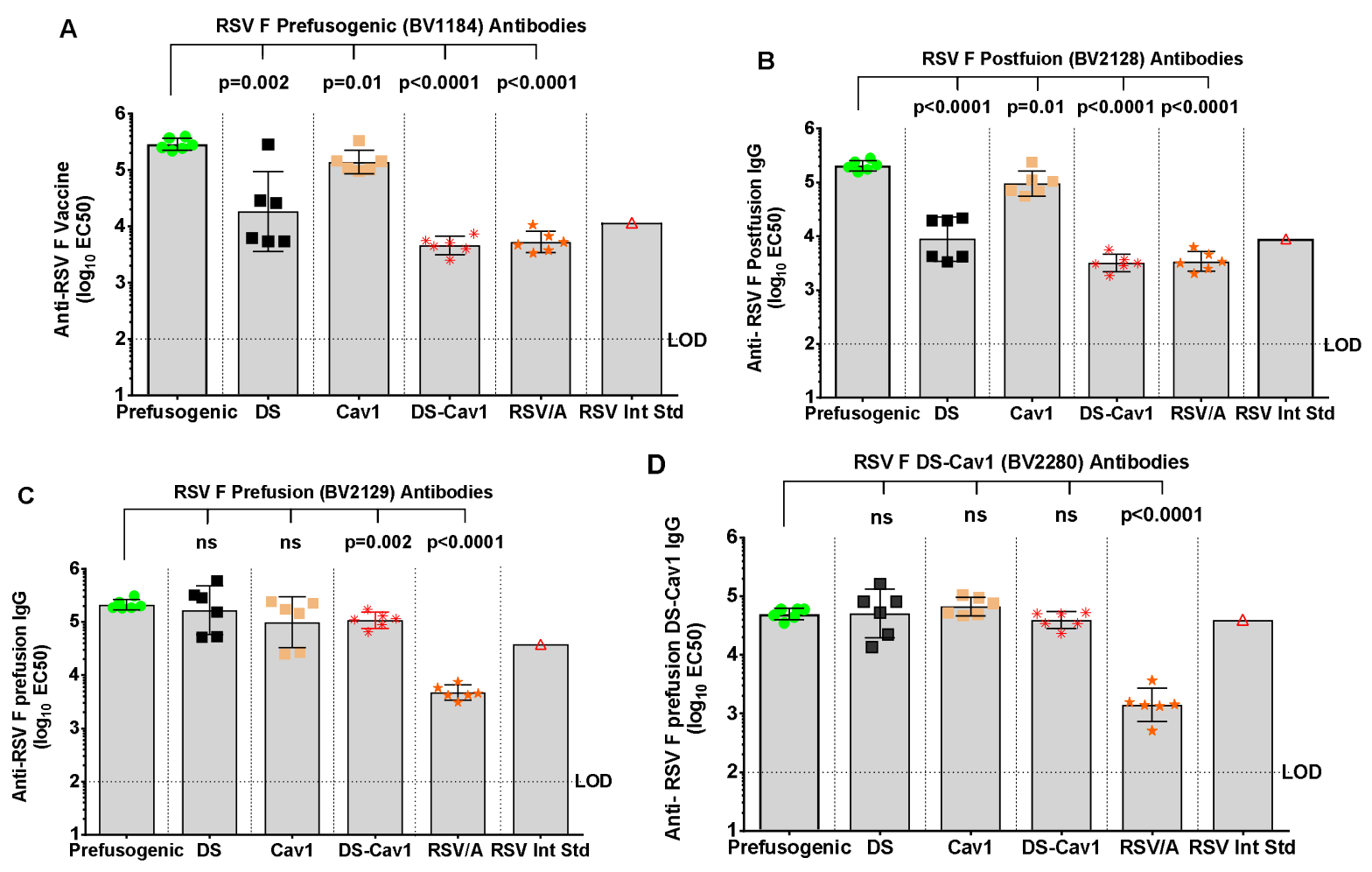
Figure 4. Immunogenicity of prefusogenic $\mathrm{F}$ and $\mathrm{F}$ variants in cotton rats. Cotton rats were immunized by intramuscular injection with $1.0 \mu \mathrm{g}$ of prefusogenic F vaccine (BV1184), DS (BV2267), Cav1 (BV2279), DSCav1 (BV2280) or postfusion F (BV2128) with $30 \mu \mathrm{g}$ aluminum phosphate on days 0 and 21. A comparative control group was intranasal challenged with $10^{6} \mathrm{pfu}$ RSV/A Long. Serum was collected for analysis 21-days after the second immunization (day 42). (A) Anti-prefusogenic F vaccine IgG (BV1184). (B) Anti-postfusion F IgG (BV2128). (C) Anti-prefusion F IgG (BV2129). (D) Anti-DS F IgG (BV2280). Not significant (ns). Horizontal bars indicated the GMT and error bars indicate the $\pm 95 \% \mathrm{CI}$. Statistical significance between the prefusogenic F (BV1184) compared to paired groups. Limit of detection (LOD). Not significant (ns).

\subsection{Prefusion and Postfusion F Antibody Specificity}

A competitive ELISA was used to determine the specificity of antibodies to prefusion and posfusion $\mathrm{F}$ conformers. Competition with prefusion F (BV2129) resulted in a 288- to 468-fold reduction in prefusionspecific antibodies in immune serum from animals immunized with DS or DS-Cav1 while competition with postfusion F (BV2128) resulted in only a <3-fold reduction in antibody levels, consistent with the majority of antibodies being directed to prefusion F structure (Figure 5A). In contrast, animals immunized with prefusogenic $\mathrm{F}$ or Cav1 produced antibodies that competed with both prefusion and postfusion F structures. Competition with prefusion resulted in 34-fold reduction in prefusion-specific $\mathrm{F}$ antibodies while competition with postfusion $\mathrm{F}$ resulted in a 13-fold reduction in antibodies, which indicates prefusogenic F and Cav1 elicited a mix of antibodies that bound both pre- and postfusion F structures (Figure 5A).

We next determined the conformation specificity of RSV/A neutralizing antibodies elicited by immunization with prefusogenic $\mathrm{F}$ compared to immunization with the prefusion $\mathrm{F}$ variants. Competition with prefusion F (BV2129) resulting in complete adsorption of neutralizing antibodies in the serum of cotton rats immunized with prefusogenic F, DS, and DS-Cav1 and near complete adsorption of neutralizing antibodies in serum of Cav1 immunized animals (Figure 5B). In contrast, adsorption with postfusion F (BV2128) resulted in partial or no loss of neutralizing antibodies. These results indicate that immunization with prefusogenic F, DS, Cav1, and DS-Cav1 elicited neutralizing antibodies that primarily recognize the prefusion F structure (Figure 5B).
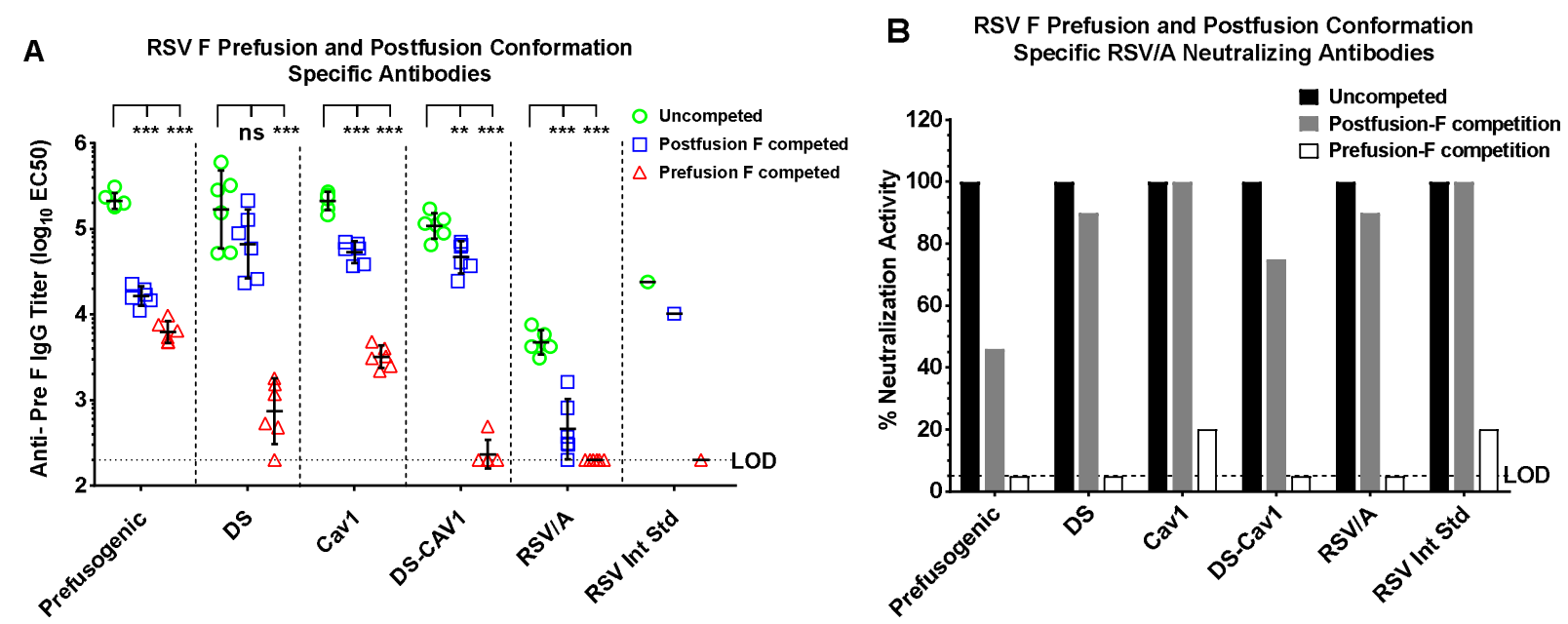

Figure 5. Prefusion and postfusion F specificity of antibodies elicited by prefusogenic F and prefusion F variants. Cotton rats were immunized and serum analyzed 21 days after the second immunization as 
described in Figure 4. (A) A competitive ELISA was used to determine the level of antibodies targeting prefusion F (BV2129) and postfusion F (BV2128) in serum of immunized cotton rats. (B) Conformationspecific neutralizing antibodies in serum competed with prefusion (BV2129) or postfusion F (BV2128). The horizontal bars indicate the group ( $\mathrm{N}=6$ per group) geometric mean titer (GMT) and the error bars indicate the $95 \% \mathrm{CI}$. Lower limit of detection (LOD). ${ }^{* *} \mathrm{p}=0.003,{ }^{* * *} \mathrm{p}<0.0001$, not significant (ns).

\subsection{RSV F Antigenic Site-specific Antibodies}

We next determined the specificity of the humoral response to key RSV F neutralizing epitopes with a panel of neutralizing mAbs in a competitive ELISA. Animals immunized with prefusion F DS, Cav1 or DS-Cav1 had significantly higher levels $(\mathrm{p} \leq 0.001$ ) of antibodies competitive with D25 (GMT $=40.8-76.2$

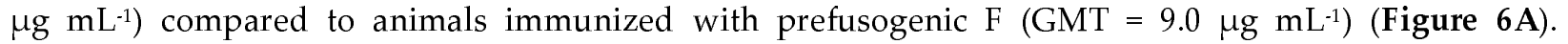
Importantly, animals immunized with prefusogenic $F$ or Cav1 had higher levels $(p<0.0001)$ of antibodies competitive with palivizumab (site II) and R1.42 (site IV). In contrast, animals immunized with prefusion DS or DS-Cav1 had significantly lower $(\mathrm{p} \leq 0.0001)$ levels of antibodies that were competitive with palivizumab (site II), R1.42 (site IV) or p27 (Figure 6B, 6C, and 6D). These results are consistent with the mouse immunogenicity result and support the hypothesis that the flexible native prefusogenic $\mathrm{F}$ elicits antibodies that recognize neutralizing epitopes on prefusion and postfusion F conformations, while prefusion variants DS and DS-Cav1 produced antibodies primarily targeting prefusion site $\varnothing$ but little or no antibodies to other neutralizing sites (II and IV).

A

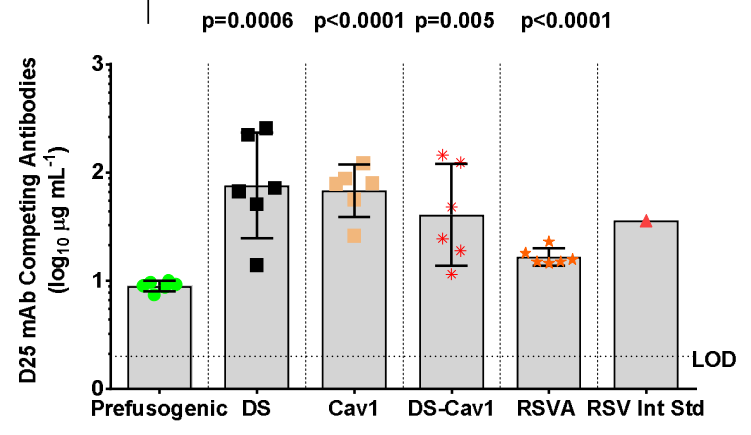

C
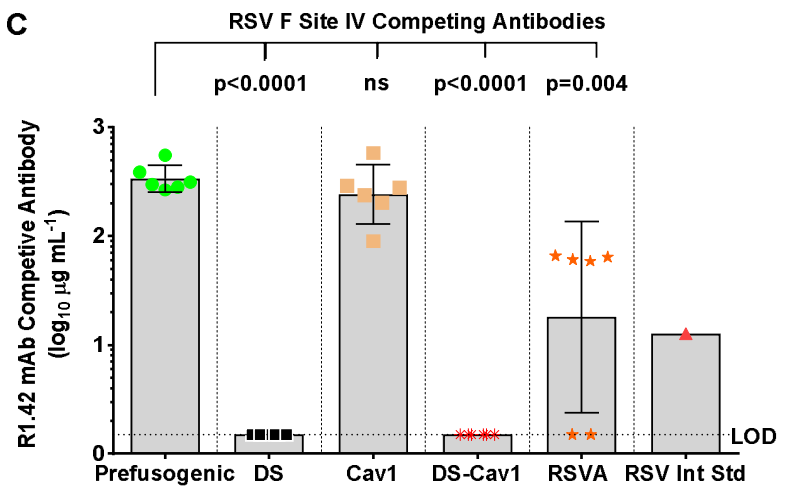

B
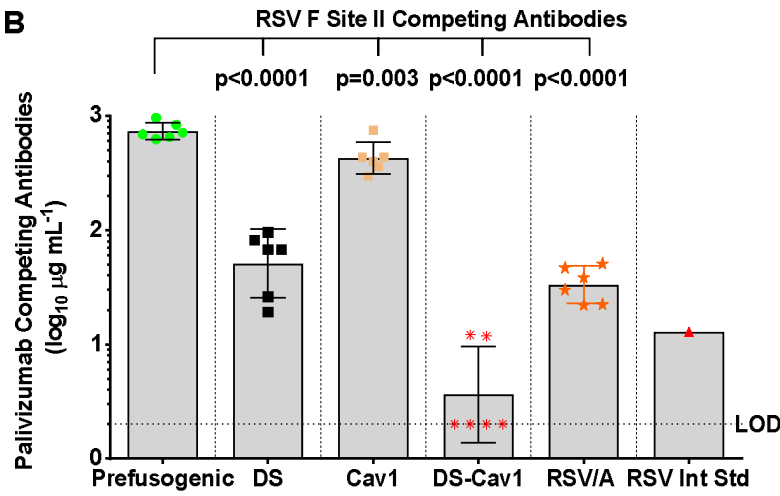

D

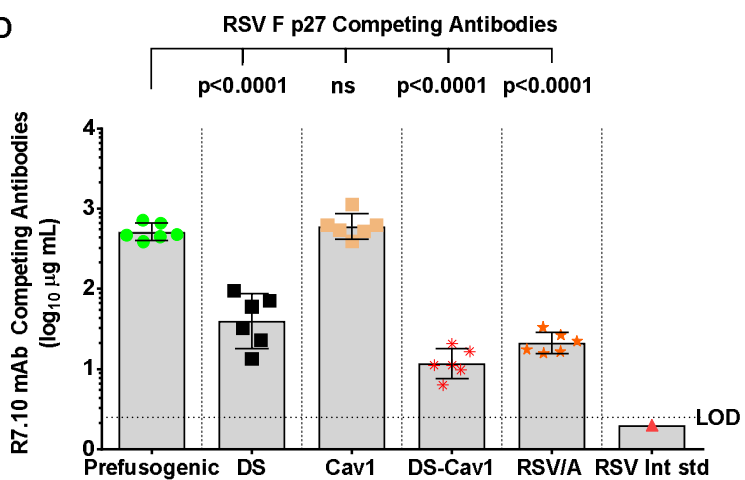

Figure 6. Specificity of antibodies elicited by prefusogenic F compared to prefusion F variants in cotton rats. Female cotton rats were immunized with $1.0 \mu \mathrm{g}$ RSV F proteins with $30 \mu \mathrm{g}$ aluminum phosphate and a control group was intranasal challenged with RSV/A. Cotton rats were immunized and serum analyzed 21 days after the second immunization as described in Figure 4. The specificity of the humoral response was determined by the competitive antibody binding ELISA using site-specific mAbs: (A) D25 
(site Ø), (B) palivizumab (site II), (C) R1.42 (site IV), and (D) R7.10 (p27). The horizontal bars indicate the group ( $\mathrm{N}=6$ per group) GMT and the error bars indicate the $\pm 95 \%$ IC. Statistical significance between the RSV prefusogenic F group (BV1184) and the paired groups is indicated. WHO RSV International Standard (RSV Int Std). Limit of detection (LOD). Not significant (ns).

\subsection{Neutralizing Antibodies and Protection against RSVIA Challenge in Cotton Rats}

RSV A and B subtype cross neutralizing antibody titers were determined in cotton rats immunized with prefusogenic $\mathrm{F}$ and prefusion $\mathrm{F}$ variants. RSV/A neutralizing antibody titers were significantly higher $(\mathrm{p} \leq 0.04)$ in animals immunized with prefusogenic $\mathrm{F}$ or Cav1 (GMT $=2,346$ and 1,594 IU mL $\mathrm{mL}^{-1}$ respectively) compared to animals immunized with DS or DS-Cav1 (GMT $=532$ and $282 \mathrm{IU} \mathrm{mL}^{-1}$, respectively) (Figure 7A). Significantly, animals immunized with prefusogenic F and Cav1 had 13- to 20fold higher levels of neutralizing antibodies that cross-neutralized RSV/B (18537) subtype (GMT = 1559 and $1264 \mathrm{IU} \mathrm{mL}^{-1}$, respectively) while animals immunized with DS or DS-Cav1 had significantly lower $(\mathrm{p} \leq 0.001)$ levels of RSV/B cross neutralizing antibody titers $\left(\mathrm{GMT}=116 \mathrm{IU} \mathrm{mL}^{-1}\right.$ and $\mathrm{GMT}=79 \mathrm{IU} \mathrm{mL}^{-1}$, respectively) (Figure 7B).

To assess the protective effects of the F proteins, immunized cotton rats were challenged 3 weeks after 2 immunization by intranasal challenge with $10^{5} \mathrm{pfu}$ RSV/A Long. Virus load was significantly lower $(\mathrm{p} \leq 0.005)$ nose homogenates of all vaccinated animals $\left(\mathrm{GMT}=1,077-14,024 \mathrm{pfu} \mathrm{g}^{-1}\right)$ compared to non-immunized animals (GMT $=558,308 \mathrm{pfu} \mathrm{g}^{-1}$ ). Furthermore, animals immunized with prefusogenic $\mathrm{F}$ had significantly lower $(\mathrm{p}<0.01)$ virus load in nasal homogenates compared to animals immunized with the DS-Cav1 (GMT $=2587 \mathrm{pfu} \mathrm{g}^{-1}$ vs. GMT $=14,024 \mathrm{pfu}^{-1}$, respectively). Nasal virus loads were not significantly in animals immunized with prefusogenic F, DS or Cav1 GMT $=1077-9290$ pfu g-1) (Figure 7C). Virus load in lung homogenates were significantly lower $(p<0.0001)$ in lungs of animals immunized with RSV F proteins were below the limit of detection (LOD $<100 \mathrm{pfu}^{-1}$ ) compared to placebo treated animals (GMT $=449,018$ pfu g$^{-1}$ ) (Figure 7D). Animals immunized with RSV F proteins had significantly reduced upper and lower respiratory tract virus load compared to non-immunized cotton rats. 

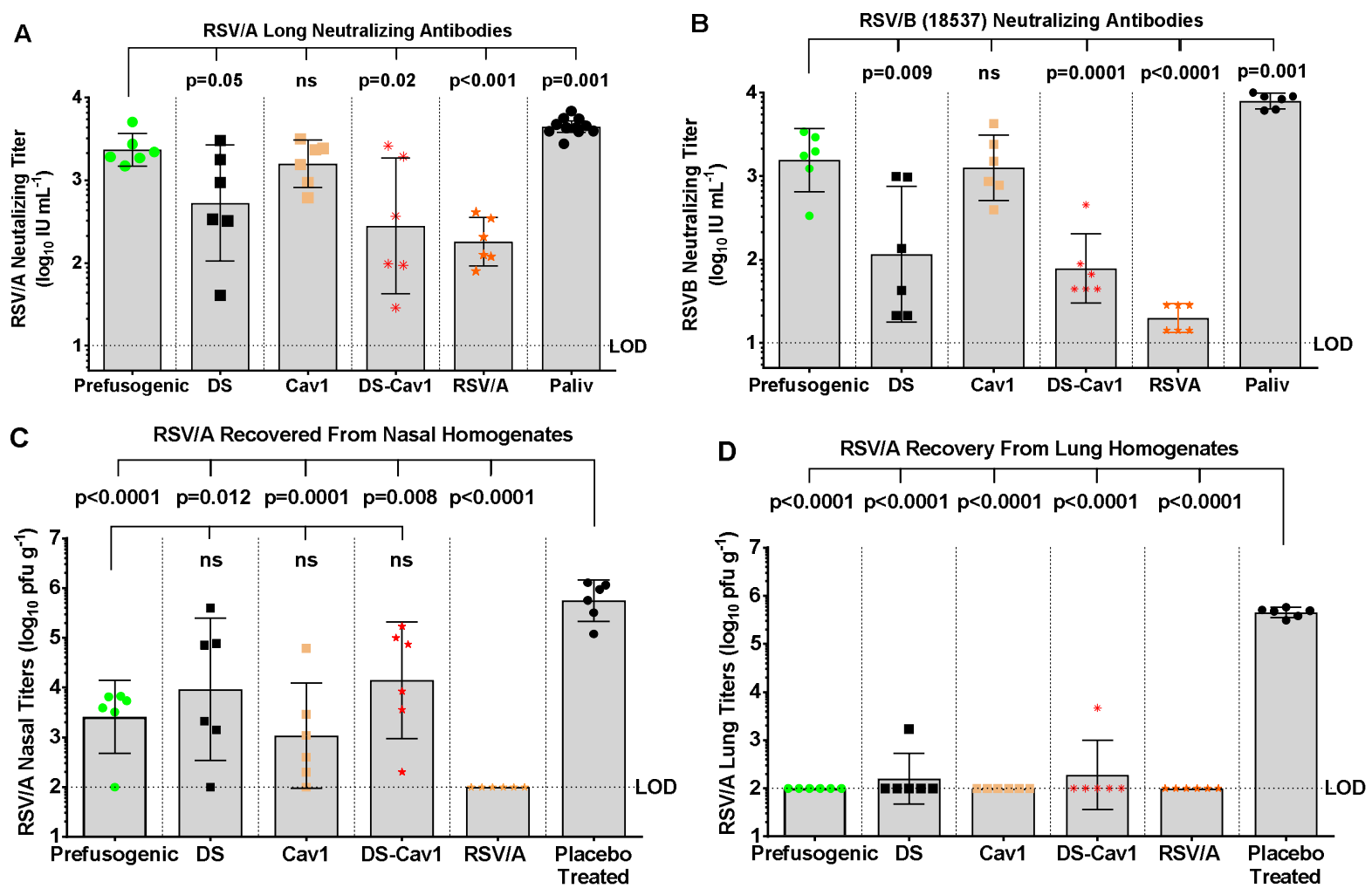

Figure 7. RSV A and B subtype neutralizing antibodies and protection against RSV challenge in cotton rats. (A, B) RSV A and B subtype cross-neutralizing antibody titers in animals immunized with prefusogenic F and prefusion F DS, Cav1 and DS-Cav1. (C, D) Nose and lung tissues were collected 4days post-challenge (day 46) and infectious virus determined with a plaque assay. Individual animal antibody titers and virus load in nasal and lung homogenates are plotted. The horizontal bars indicate the group GMT and error bars indicate the $\pm 95 \%$ CI. Statistical significance between paired groups is indicated by brackets. Limit of detection (LOD). Not significant (ns).

\section{Discussion}

Prefusion stabilized F DS-Cav1 has been proposed to be a potentially superior vaccine candidate since high affinity neutralizing antibodies bind to conformation-dependent sites $\varnothing$ and $\mathrm{V}[22,25,35]$, although there is no clinical evidence to support the assumption that prefusion-specific immunity alone is protective against RSV A and B subtypes [24]. There is however considerable clinical and preclinical evidence that multiple F-specific neutralizing epitopes contribute to protection. For instance, passive immunization of high-risk infants with $\mathrm{mAbs}$ palivizumab or motavizumab targeting antigenic site II is protective against developing RSV-LRTI in high-risk infants [3,4,37]. Infants born with maternal anti-F antibodies to site II develop less severe RSV-related disease compared to infants without maternal antibodies [38-40]. Further, we have also shown that infant baboons born to mothers' immunization with prefusogenic F are substantially protected against developing RSV-LRTI compared to infants born to non-immunized mothers [32]. We have shown that women of childbearing age and older adults immunized with near full-length prefusogenic F nanoparticles produce antibodies that bind site II $[33,34,41]$. 
Here, we compared the humoral immune response elicited by native prefusogenic $\mathrm{F}$ to prefusion $\mathrm{F}$ variants. Using flexible prefusogenic F backbone, prefusion stable variants DS, Cav1 and DS-Cav1 were generated. Mice and cotton rats immunized with DS or DS-Cav1 had significant levels of antibodies targeting prefusion-specific sites $\varnothing$ and V (Figure 3C, Figure 6A and Supplementary Figure S1) but had little or no antibodies to key neutralizing antigenic sites II or IV (Figure 3D, 3E and Figure 6B, 6C). Immunization with prefusogenic $\mathrm{F}$ also elicited elevated levels of antibodies competitive with D25, although at lower levels compared to the prefusion variants (Figure 6A). In contrast, immunization with the flexible prefusogenic $\mathrm{F}$ elicited antibodies that recognized prefusion and postfusion $\mathrm{F}$ structures (Figure 5A). Importantly, immunization with prefusogenic F produced antibodies that broadly crossneutralized RSV A and B subtypes while immunization with prefusion DS or DS-Cav1 elicited antibodies that were RSV/A neutralizing and little RSV/B cross-neutralizing antibodies (Figure 7A and 7B). Collectively, these results show that prefusogenic $\mathrm{F}$ was significantly more immunogenic and the flexible structure allowed B-cell access to important neutralizing epitopes that were not available more ridged $\mathrm{F}$ structures.

The concept of type 1 trimer breathing, dissociation, and monomerization on the lipid bilayer has been described for the HIV envelope (Env) glycoprotein gp120 and gp41. Lee et al [42-44] have shown that antibody binding to flexible Env gp promotes the dissociation of the Env gp120/gp40, which promotes trimer instability and may be an unrecognized mechanism of protection. These observations support the concept that Env immunogens that closely mimic the flexible Env gp structure may be critical considerations in vaccine design and allows accessibility of B-cells to Env epitopes that promote trimer instability and decay. This same mechanism may also be important consideration for design of RSV F trimeric vaccines. Gillman et al. [19] recently reported that CR9501 mAb binding to RSV F at a specific angle promotes trimer disassembly within the lipid bi-layer. These observations suggest that vaccine strategies targeting type 1 trimers should consider flexible trimeric structures and the plasticity of humoral immunity. Such vaccine strategies may be superior to ridged structures.

\section{Conclusions}

In this report, we hypothesized trimeric vaccine strategies that are based on breathable $\mathrm{F}$ structures, and provide accessibility of B-cells to epitopes on intermediate conformations, may collectively contribute to broad protective immunity, while less flexible F structures restrict B-cell access to important protective epitopes. We compared the humoral immune responses to prefusogenic $F$ to prefusion stabilized variants. Native prefusogenic F was significantly more immunogenic and elicited antibodies to conformation-dependent and conformation-independent epitopes that broadly cross neutralized RSV A and B subtypes while prefusion variants were less immunogenic and elicited antibodies primarily targeting prefusion $\mathrm{F}$ structure. These results have implications for vaccine strategies targeting other type 1 integral membrane proteins. 
Supplementary Materials: Appendix A Figure S1. Competitive binding of RSV F site-specific monoclonal antibodies with immune serum determined by bio-layer interferometry (BLI) in cotton rats.

Author Contributions: Authors NP, JHT, RF, KJ, MW, AP, MGX, HL, and MJM contributed to conceptualization of experiments, generation of data and analysis, and interpretation of the data. Authors GG, LE and GS, contributed to drafting, making critical revisions, and giving approval for submission.

Funding: Support of this work was provided by Novavax, Inc. The funder had no role in the study design, data collection and analysis, decision to publish, or preparation of the manuscript.

Acknowledgments: The authors wish to thank James Norton for upstream cell culture technical support, Rafia Khatoon for producing the plasmid constructs, Malgorzata Wisniewska for plaque production and preparation of baculovirus stocks, Will Moffitt for downstream purification of RSV F proteins and Sonai Maciejewski for preforming serological assessments.

Conflicts of Interest: Authors NP, JHT, RF, KJ, MW, AP, MGX, HL, MJM, GG, LE, and GS are current or past employees of Novavax, Inc, a for-profit organization, and these authors own stock or hold stock options. These interests do not alter the authors adherence to policies on sharing data and materials.

\section{References}

1. Shi T, McAllister DA, O'Brien KL, Simoes EAF, Madhi SA, Gessner BD, Polack FP, Balsells E, Acacio $\mathrm{S}$, Aguayo C, et al. Global, regional, and national disease burden estimates of acute lower respiratory infections due to respiratory syncytial virus in young children in 2015: a systematic review and modelling study. Lancet. 2017, 390, 946-958. https://doi:10.1016/S0140-6736(17)30938-8.

2. Shi T, Denouel A, Tietjen AK, Campbell I, Moran E, Li X, Campbell H, Demont C, Nyawanda BO, Chu HY, Stoszek SK, Krishnan A, Openshaw P, Falsey AR, Nair H; RESCEU Investigators. Global Disease Burden Estimates of Respiratory Syncytial Virus-Associated Acute Respiratory Infection in Older Adults in 2015: A Systematic Review and Meta-Analysis. J Infect Dis. 2019, pii: jiz059. https://doi:10.1093/infdis/jiz059.

3. Johnson S, Oliver C, Prince GA, Hemming VG, Pfarr DS, Wang SC, Dormitzer M, O'Grady J, Koenig S, Tamura JK, Woods R, Bansal G, Couchenour D, Tsao E, Hall WC, Young JF. Development of a humanized monoclonal antibody (MEDI-493) with potent in vitro and in vivo activity against respiratory syncytial virus. J Infect Dis. 1997, 176, 1215-1224.

4. Palivizumab, a humanized respiratory syncytial virus monoclonal antibody, reduces hospitalization from respiratory syncytial virus infection in high-risk infants. The IMpact-RSV Study Group. Pediatrics. 1998, 102, 531-537.

5. Del Vecchio A, Franco C, Del Vecchio K, Umbaldo A, Capasso L, Raimondi F. RSV prophylaxis in premature infants. Minerva Pediatr. 2018, 70, 579-588. https://doi:10.23736/S0026-4946.18.05300-8.

6. Hause AM, Henke DM, Avadhanula V, Shaw CA, Tapia LI, Piedra PA. Sequence variability of the respiratory syncytial virus (RSV) fusion gene among contemporary and historical genotypes of RSV/A and RSV/B. PLoS One. 2017, 12, e0175792. https://doi:10.1371/journal.pone.0175792. 
7. Hicks SN, Chaiwatpongsakorn S, Costello HM, McLellan JS, Ray W, Peeples ME. Five Residues in the Apical Loop of the Respiratory Syncytial Virus Fusion Protein F2 Subunit Are Critical for Its Fusion Activity. J Virol. 2018, 92, e00621-18. https://doi:10.1128/JVI.00621-18.

8. McLellan JS. Neutralizing epitopes on the respiratory syncytial virus fusion glycoprotein. Curr Opin Virol. 2015, 11, 70-75. https://doi:10.1016/j.coviro.2015.03.002.

9. Swanson KA, Settembre EC, Shaw CA, Dey AK, Rappuoli R, Mandl CW, Dormitzer PR, Carfi A. Structural basis for immunization with postfusion respiratory syncytial virus fusion $\mathrm{F}$ glycoprotein (RSV F) to elicit high neutralizing antibody titers. Proc Natl Acad Sci U S A. 2011, 108, 9619-9624. https://doi:10.1073/pnas.1106536108.

10. Fuentes S, Coyle EM, Beeler J, Golding H, Khurana S. Antigenic Fingerprinting following Primary RSV Infection in Young Children Identifies Novel Antigenic Sites and Reveals Unlinked Evolution of Human Antibody Repertoires to Fusion and Attachment Glycoproteins. PLoS Pathog. 2016, 12, e1005554. https://doi:10.1371/journal.ppat.1005554.

11. Begoña Ruiz-Argüello M, González-Reyes L, Calder LJ, Palomo C, Martín D, Saíz MJ, García-Barreno B, Skehel JJ, Melero JA. Effect of proteolytic processing at two distinct sites on shape and aggregation of an anchorless fusion protein of human respiratory syncytial virus and fate of the intervening segment. Virology. 2002, 298, 317-326.

12. González-Reyes L, Ruiz-Argüello MB, García-Barreno B, Calder L, López JA, Albar JP, Skehel JJ, Wiley DC, Melero JA. Cleavage of the human respiratory syncytial virus fusion protein at two distinct sites is required for activation of membrane fusion. Proc Natl Acad Sci U S A. 2001, 98, 98599864.

13. Bolt G, Pedersen LO, Birkeslund HH. Cleavage of the respiratory syncytial virus fusion protein is required for its surface expression: role of furin. Virus Res. 2000, 68, 25-33.

14. Scheid A, Choppin PW. Two disulfide-linked polypeptide chains constitute the active F protein of paramyxoviruses. Virology. 1977, 80, 54-66.

15. Schlender J, Zimmer G, Herrler G, Conzelmann KK. Respiratory syncytial virus (RSV) fusion protein subunit F2, not attachment protein G, determines the specificity of RSV infection. J Virol. 2003, 77, 4609-4616.

16. Lawlor HA, Schickli JH, Tang RS. A single amino acid in the F2 subunit of respiratory syncytial virus fusion protein alters growth and fusogenicity. $J$ Gen Virol. 2013, 94, 2627-2635. https://doi:10.1099/vir.0.055368-0.

17. Krarup A, Truan D, Furmanova-Hollenstein P, Bogaert L, Bouchier P, Bisschop IJM, Widjojoatmodjo MN, Zahn R, Schuitemaker H, McLellan JS, Langedijk JPM. A highly stable prefusion RSV F vaccine derived from structural analysis of the fusion mechanism. Nat Commun. 2015, 6, 8143. https://doi:10.1038/ncomms9143.

18. Bermingham IM, Chappell KJ, Watterson D, Young PR. The Heptad Repeat C Domain of the Respiratory Syncytial Virus Fusion Protein Plays a Key Role in Membrane Fusion. J Virol. 2018, 92, pii: e01323-17. https://doi:10.1128/JVI.01323-17. 
19. Gilman MSA, Furmanova-Hollenstein P, Pascual G, B van 't Wout A, Langedijk JPM, McLellan JS. Transient opening of trimeric prefusion RSV F proteins. Nat Commun. 2019, 10, 2105. https://doi:10.1038/s41467-019-09807-5.

20. Calder LJ, González-Reyes L, García-Barreno B, Wharton SA, Skehel JJ, Wiley DC, Melero JA. Electron microscopy of the human respiratory syncytial virus fusion protein and complexes that it forms with monoclonal antibodies. Virology. 2000, 271, 122-131.

21. Yunus AS, Jackson TP, Crisafi K, Burimski I, Kilgore NR, Zoumplis D, Allaway GP, Wild CT, Salzwedel K. Elevated temperature triggers human respiratory syncytial virus $\mathrm{F}$ protein six-helix bundle formation. Virology. 2010, 396, 226-237. https://doi:10.1016/j.virol.2009.10.040.

22. McLellan JS, Chen M, Leung S, Graepel KW, Du X, Yang Y, Zhou T, Baxa U, Yasuda E, Beaumont T, Kumar A, Modjarrad K, Zheng Z, Zhao M, Xia N, Kwong PD, Graham BS. Structure of RSV fusion glycoprotein trimer bound to a prefusion-specific neutralizing antibody. Science. 2013, 340, 1113-1117. https://doi:10.1126/science.1234914.

23. McLellan JS, Chen M, Joyce MG, Sastry M, Stewart-Jones GB, Yang Y, Zhang B, Chen L, Srivatsan S, Zheng A, Zhou T, Graepel KW, Kumar A, Moin S, Boyington JC, Chuang GY, Soto C, Baxa U, Bakker AQ, Spits H, Beaumont T, Zheng Z, Xia N, Ko SY, Todd JP, Rao S, Graham BS, Kwong PD. Structurebased design of a fusion glycoprotein vaccine for respiratory syncytial virus. Science. 2013, 342, 592598. https://doi:10.1126/science.1243283.

24. Crank MC, Ruckwardt TJ, Chen M, Morabito KM, Phung E, Costner PJ, Holman LA, Hickman SP, Berkowitz NM, Gordon IJ, Yamshchikov GV, Gaudinski MR, Kumar A, Chang LA, Moin SM, Hill JP, DiPiazza AT, Schwartz RM, Kueltzo L, Cooper JW, Chen P, Stein JA, Carlton K, Gall JG, Nason MC, Kwong PD, Chen GL, Mascola JR, McLellan JS, Ledgerwood JE, Graham BS; VRC 317 Study Team. A proof of concept for structure-based vaccine design targeting RSV in humans. Science. 2019, 365, 505509. https://doi:10.1126/science.aav9033.

25. Ngwuta JO, Chen M, Modjarrad K, Joyce MG, Kanekiyo M, Kumar A, Yassine HM, Moin SM, Killikelly AM, Chuang GY, Druz A, Georgiev IS, Rundlet EJ, Sastry M, Stewart-Jones GB, Yang Y, Zhang B, Nason MC, Capella C, Peeples ME, Ledgerwood JE, McLellan JS, Kwong PD, Graham BS. Prefusion F-specific antibodies determine the magnitude of RSV neutralizing activity in human sera. Sci Transl Med. 2015, 7, 309ra162. https://doi:10.1126/scitranslmed.aac4241.

26. Jones HG, Battles MB, Lin CC, Bianchi S, Corti D, McLellan JS. Alternative conformations of a major antigenic site on RSV F. PLoS Pathog. 2019, 15, e1007944. https://doi:10.1371/journal.ppat.1007944.

27. Corti D, Bianchi S, Vanzetta F, Minola A, Perez L, Agatic G, Guarino B, Silacci C, Marcandalli J, Marsland BJ, Piralla A, Percivalle E, Sallusto F, Baldanti F, Lanzavecchia A. Cross-neutralization of four paramyxoviruses by a human monoclonal antibody. Nature. 2013, 50, 439-443. https://doi:10.1038/nature12442.

28. Xie Q, Wang Z, Ni F, Chen X, Ma J, Patel N, Lu H, Liu Y, Tian JH, Flyer D, Massare MJ, Ellingsworth L, Glenn G, Smith G, Wang Q. Structure basis of neutralization by a novel site II/IV antibody against respiratory syncytial virus fusion protein. PLoS One. 2019, 14, e0210749. https://doi:10.1371/journal.pone.0210749. 
29. Smith G, Raghunandan R, Wu Y, Liu Y, Massare M, Nathan M, Zhou B, Lu H, Boddapati S, Li J, Flyer D, Glenn G. Respiratory syncytial virus fusion glycoprotein expressed in insect cells form protein nanoparticles that induce protective immunity in cotton rats. PLoS One. 2012, 7, e50852. https://doi:10.1371/journal.pone.0050852.

30. McLellan JS, Yang Y, Graham BS, Kwong PD. Structure of respiratory syncytial virus fusion glycoprotein in the postfusion conformation reveals preservation of neutralizing epitopes. $J$ Virol. 2011, 85, 7788-7796. https://doi:10.1128/JVI.00555-11.

31. Patel N, Massare MJ, Tian JH, Guebre-Xabier M, Lu H, Zhou H, Maynard E, Scott D, Ellingsworth L, Glenn G, Smith G. Respiratory syncytial virus prefusogenic fusion $(F)$ protein nanoparticle vaccine: Structure, antigenic profile, immunogenicity, and protection. Vaccine. 2019, 37, 6112-6124. https://doi:10.1016/j.vaccine.2019.07.089.

32. Welliver RC, Papin JF, Preno A, Ivanov V, Tian JH, Lu H, Guebre-Xabier M, Flyer D, Massare MJ, Glenn G, Ellingsworth L, Smith G. Maternal immunization with RSV fusion glycoprotein vaccine and substantial protection of neonatal baboons against respiratory syncytial virus pulmonary challenge. Vaccine. 2019, 38, 1258-1270. https://doi:10.1016/j.vaccine.2019.11.003.

33. Glenn GM, Fries LF, Thomas DN, Smith G, Kpamegan E, Lu H, Flyer D, Jani D, Hickman SP, Piedra PA.. A randomized, blinded, controlled, dose-ranging study of a respiratory syncytial virus recombinant fusion $(\mathrm{F})$ nanoparticle vaccine in healthy women of childbearing age. J Infect Dis. 2016, 213, 411-422. https://doi.org/10.1093/infdis/jiv406.

34. Fries L, Shinde V, Stoddard JJ, Thomas DN, Kpamegan E, Lu H, Smith G, Hickman SP, Piedra P, Glenn GM. Immunogenicity and safety of a respiratory syncytial virus fusion protein (RSV F) nanoparticle vaccine in older adults. Immun Ageing. 2017, 14, 8. https://doi.org/10.1186/s12979-0170090-7.

35. Mousa JJ, Kose N, Matta P, Gilchuk P, Crowe JE Jr. A novel pre-fusion conformation-specific neutralizing epitope on the respiratory syncytial virus fusion protein. Nat Microbiol. 2017, 2:16271. https://doi:10.1038/nmicrobiol.2016.271.

36. Raghunandan R, Lu H, Zhou B, Xabier MG, Massare MJ, Flyer DC, Fries LF, Smith GE, Glenn GM. An insect cell derived respiratory syncytial virus (RSV) F nanoparticle vaccine induces antigenic site II antibodies and protects against RSV challenge in cotton rats by active and passive immunization. Vaccine. 2014, 32, 6485-6492. https://doi:10.1016/j.vaccine.2014.09.030.

37. Beeler JA, van Wyke Coelingh K. Neutralization epitopes of the F glycoprotein of respiratory syncytial virus: effect of mutation upon fusion function. J Virol. 1989, 63, 2941-2950.

38. de Sierra TM, Kumar ML, Wasser TE, Murphy BR, Subbarao EK. Respiratory syncytial virus-specific immunoglobulins in preterm infants. J Pediatr. 1993, 122, 787-791.

39. Lamprecht CL, Krause HE, Mufson MA. Role of maternal antibody in pneumonia and bronchiolitis due to respiratory syncytial virus. J Infect Dis. 1976, 134, 211-217.

https://doi.org/10.1093/infdis/134.3.211. 
40. Roca A, Abacassamo F, Loscertales MP, Quintó L, Gómez-Olivé X, Fenwick F, Saiz JC, Toms G, Alonso PL. Prevalence of respiratory syncytial virus IgG antibodies in infants living in a rural area of Mozambique. J Med Virol. 2002, 67, 616-623.

41. August A, Glenn GM, Kpamegan E, Hickman SP, Jani D, Lu H, Thomas DN, Wen J, Piedra PA, Fries LF. A phase 2 randomized, observer-blind, placebo-controlled, dose-ranging trial of aluminumadjuvanted respiratory syncytial virus $\mathrm{F}$ particle vaccine formulations in healthy women of childbearing age. Vaccine. 2017, 35, 3749-3759. https://doi.org/10.1016/j.vaccine.2017.05.045.

42. Lee JH, Leaman DP, Kim AS, Torrents de la Peña A, Sliepen K, Yasmeen A, Derking R, Ramos A, de Taeye SW, Ozorowski G, Klein F, Burton DR, Nussenzweig MC, Poignard P, Moore JP, Klasse PJ, Sanders RW, Zwick MB, Wilson IA, Ward AB. Antibodies to a conformational epitope on gp41 neutralize HIV-1 by destabilizing the Env spike. Nat Commun. 2015, 6, 8167. https://doi:10.1038/ncomms9167.

43. Munro JB, Gorman J, Ma X, Zhou Z, Arthos J, Burton DR, Koff WC, Courter JR, Smith AB 3rd, Kwong PD, Blanchard SC, Mothes W. Conformational dynamics of single HIV-1 envelope trimers on the surface of native virions. Science. 2014, 346, 759-763. https://doi:10.1126/science.

44. Rutten L, Lai YT, Blokland S, Truan D, Bisschop IJM, Strokappe NM, Koornneef A, van Manen D, Chuang GY, Farney SK, Schuitemaker H, Kwong PD, Langedijk JPM. A Universal Approach to Optimize the Folding and Stability of Prefusion-Closed HIV-1 Envelope Trimers. Cell Rep. 2018, 23, 584-595. https://doi:10.1016/j.celrep.2018.03.061. 